\title{
Tidal dynamics on the North Patagonian Argentinean Gulfs
}

\author{
Mariano H. Tonini ${ }^{\text {a, }}$, Elbio D. Palma ${ }^{\text {b }}$ \\ ${ }^{a}$ Centro para el Estudio de Sistemas Marinos (CESIMAR,CENPAT-CONICET), Puerto Madryn, Argentina \\ ${ }^{\mathrm{b}}$ Departamento de Física, Universidad Nacional del Sur and Instituto Argentino de Oceanografia (IADO/CONICET), Bahía Blanca, Argentina
}

\section{A R T I C L E I N F O}

\section{Article history:}

Received 8 August 2016

Received in revised form 16 February 2017

Accepted 21 February 2017

Available online 27 February 2017

\section{Keywords:}

North Patagonian gulfs

Tidal dynamics

Tidal residual currents

Topographic eddies

Vorticity analysis

\begin{abstract}
A B S T R A C T
Semidiurnal $\left(\mathrm{M}_{2}, \mathrm{~S}_{2}, \mathrm{~N}_{2}\right.$ and $\left.\mathrm{K}_{2}\right)$, diurnal $\left(\mathrm{K}_{1}, \mathrm{O}_{1}\right.$ and $\left.\mathrm{P}_{1}\right)$ and quarter-diurnal $\left(\mathrm{M}_{4}, \mathrm{MN}_{4}\right.$ and $\left.\mathrm{MS}_{4}\right)$ barotropic tides over the North Patagonian Gulfs of Argentina: San Matías, (SMG), Nuevo (NG) and San José (SJG) are examined using a regional numerical model. Detailed comparison of computed elevations and currents with those obtained from harmonic analysis of few long-term observational records showed good agreement. A large amplification of all semidiurnal waves is recorded when they cross SMG mouth. Most of the tidal energy coming from the south at this frequency dissipates to the northeast of Valdés Península, where the largest tidal currents are located. While $\mathrm{M}_{2}$ currents (up to $2-2.5 \mathrm{~m} / \mathrm{s}$ ) are dominant, there are large $S_{2}$ and $N_{2}$ currents and locally intensified diurnal currents $(5-10 \mathrm{~cm} / \mathrm{s})$ in some outer shelf locations. At difference with the semidiurnal and diurnal tides the higher harmonics $\mathrm{M}_{4}, \mathrm{MS}_{4}$ and $\mathrm{MN}_{4}$ develop a large amplification inside the NG. The model revealed a complex field of tidal residual currents. The intensity of such currents in some locations are of the same order of magnitude as those generated by winds or offshore forcing. We have identified three main patterns of residual circulation: regional coastal currents and gulf-wide gyres; inlet eddies and, topographic eddies. Vorticity balances and sensitivity experiments indicate that non-linear advection and bottom topography are the key mechanisms involved in the generation of these residual structures.
\end{abstract}

오 2017 Elsevier Ltd. All rights reserved.

\section{Introduction}

The North Patagonian Gulfs (NPGs) region comprises three semi-enclosed gulfs: the San Matias (SMG), Nuevo (NG) and San Jose (SJG) and is considered one of the most productive areas of the Patagonian Shelf ecosystem (Acha et al., 2004, Fig. 1). Two notable physical features of this region are the large amplification of the tidal wave and the localized high tidal energy dissipation rate near the SMG entrance (Glorioso and Flather, 1997; Palma et al., 2004; Simionato et al., 2004; Moreira et al., 2011). The dynamical interplay of the tidal forcing with geomorphological features like headlands, promontories, semi-enclosed areas, deep basins and narrow gulf's entrances leads to the generation of strong fronts and re-circulating patterns (gyres) that have been observed in hydrographic data and remote observations (Piola and Scasso, 1988; Gagliardini and Rivas, 2004) and crudely modeled with low resolution models (Glorioso and Simpson, 1994). Many physical and bio-geochemical processes in the area are also affected by the

\footnotetext{
* Corresponding author.

E-mail addresses: tonini@cenpat-conicet.gob.ar (M.H. Tonini), uspalma@criba. edu.ar (E.D. Palma).
}

details of tidal dynamics. For instance, tides influence the extent of vertical mixing, and thus partially determine the location of temperature fronts that are often observable from satellite imagery (Pisoni et al., 2014; Tonini et al., 2013) with potential large impacts on fisheries (Romero et al., 2006) and CO2 fluxes (Bianchi et al., 2005).

Albeit important, knowledge of the details of the tidal dynamics and tidal residual currents in this area is still unsatisfactory and largely based on low-resolution (coarser than $10 \mathrm{~km}$ ) regional models of the Patagonian Shelf (Glorioso and Flather, 1997; Palma et al., 2004; Simionato et al., 2004) and the analysis of a limited number of in situ current measurements (e.g., Rivas, 1997; Moreira et al., 2009). Two exceptions are the recent high resolution models $(\sim 1 \mathrm{~km})$ of Moreira et al. (2011) and Tonini et al. (2013). Moreira et al. (2011) studied the propagation of the $M_{2}$ tidal wave in the NPGs applying a set of three nested high resolution models based on a z-coordinate model. The work of Tonini et al. (2013) indicates that tidal forcing plays an important role in shaping not only the barotropic but also the baroclinic ocean response. Tidal forcing significantly contribute to delineate the frontal structures, and the seasonal circulation patterns in the NPGs, mainly in the summer season when the increased stratification interacts with the tides 


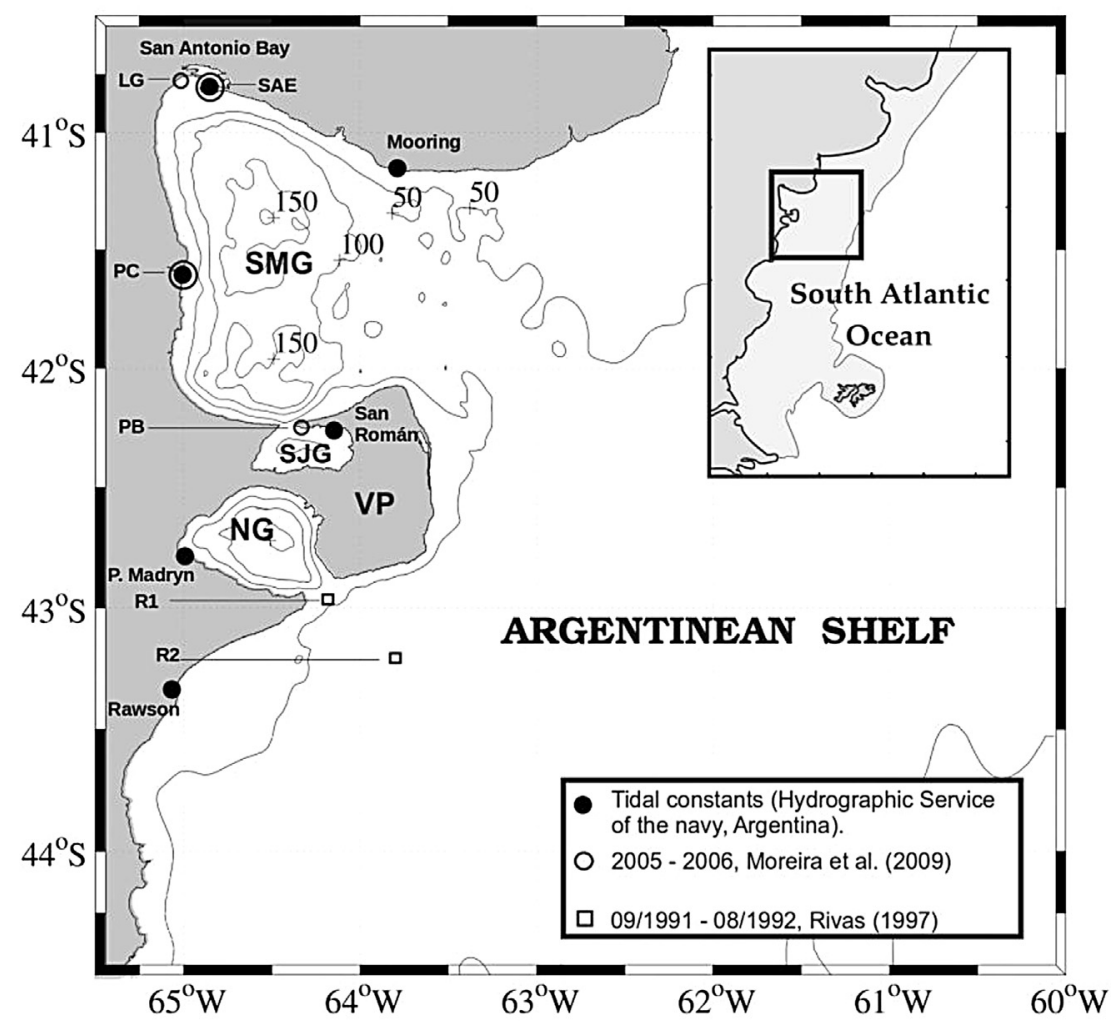

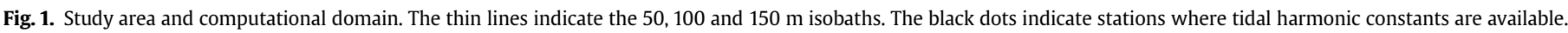
The black circles and black squares show the location of available current time-series. NG: Nuevo Gulf; SJG: San José Gulf; SMG: San Matías Gulf; VP: Valdés Península.

intensifing a cyclonic circulation inside SMG and NG.

Although much have been learned about this region with the previous work, there is still room to be learned in terms of the dynamical role played by the different tidal constituents, the tidal energy fluxes and dissipation mechanisms and the detailed analysis of the residual patterns and the physical mechanisms responsible for its shape and structure. In this article we propose to further explore the barotropic tidal dynamics in this region using a suite of high resolution numerical experiments encompassing the NPGs region based on the earlier model of Tonini et al. (2013).

\section{Data and methods}

\subsection{Model set-up}

The numerical model used in this study is the Regional Ocean Modelling System (ROMS, Shchepetkin and McWilliams, 2005). In the vertical, the primitive equations are discretized over variable topography using stretched terrain-following coordinates. In the horizontal, the primitive equations are evaluated using orthogonal curvilinear coordinates on a staggered Arakawa C-grid. For the vertical mixing parameterization we selected the scheme developed by Mellor and Yamada (1982).

The model computational grid has 271 nodes in alongshore (N$\mathrm{S})$ and 241 nodes in a cross-shore (W-E) direction and extends from $40^{\circ}$ to $44^{\circ} \mathrm{S}$ and from $60^{\circ}$ to $65^{\circ} \mathrm{W}$ (Fig. 1 ). The spatial resolution of the grid is variable, with maximum resolution $(\sim 1 \mathrm{~km})$ in the gulfs. In the vertical the model equations are discretized in 20 s-levels, with higher vertical resolution at the top and bottom layers. The bathymetry is based on digitized nautical charts. The model has three open boundaries (Fig. 1) where a combination of the Flather scheme for the barotropic mode and the Orlanski radiation condition for the internal velocities is used (Marchesiello et al. 2001). At these lateral open boundaries we imposed tidal amplitudes and phases of 8 principal constituents ( 4 semidiurnal, $M_{2}, S_{2}$, $\mathrm{N}_{2}, \mathrm{~K}_{2}$, and four diurnal $\mathrm{K}_{1}, \mathrm{O}_{1}, \mathrm{P}_{1}$ and $\left.\mathrm{Q}_{1}\right), 2$ long-term constituents $\left(\mathrm{M}_{\mathrm{f}}\right.$ and $\left.\mathrm{M}_{\mathrm{m}}\right)$ and 3 higher harmonics $\left(\mathrm{M}_{4}, \mathrm{MS}_{4}\right.$ and $\left.\mathrm{MN}_{4}\right)$ interpolated from a global tidal model (TPXO6, Egbert et al., 1994).

\subsection{Observational data}

For validating the model results we employ tidal amplitudes and phases from 5 coastal stations of the National Navy Hydrographic Service with record-length larger than 30 days, one recently deployed tidal gauge with record-length larger than a year (Lago et. al, 2017, under review, Mooring in Table 1) and the scarce available in-situ current observations (Fig. 1). The in situ observations consist in six current meter data from two different sources: the two stations south of domain (R1 and R2) were collected in a cross-shelf section near $43^{\circ} \mathrm{S}$ between September 1991 and August 1992, (Rivas, 1997). The other four stations are located inside SJG (Playa Bengoa, PB) and SMG (from south to north, Punta Colorada, PC, Las Grutas, LG and San Antonio Este, SAE), and the data were collected between July 2005 and December 2006 (Moreira et al., 2009). The timespan of the time series is of approximately 1 month each (see Table 2).

\section{Tidal characteristics}

We will focus here on the description, mapping and analysis of the seven major tidal constituents $\mathrm{M}_{2}, \mathrm{~S}_{2}, \mathrm{~N}_{2}, \mathrm{~K}_{2}, \mathrm{~K}_{1}, \mathrm{O}_{1}$ and $\mathrm{P}_{1}$ and the overtides $\mathrm{M}_{4}$ and $\mathrm{MS}_{4}$ and their validation with available data. Although the model has been forced by 13 tidal components only those components with maximum amplitudes larger than $2 \mathrm{~cm}$ 
inside the Gulfs are considered in detail. Beginning from a state of rest, the model is integrated forward in time during one year. Elevations and currents were then analysed using standard harmonic analysis (Foreman, 1977) to produce the co-amplitude and cophases charts, tidal ellipses, tidal energy fluxes and tidal energy dissipation maps.

\subsection{Cotidal charts}

The semidiurnal tidal waves $\left(\mathrm{M}_{2}, \mathrm{~S}_{2}, \mathrm{~N}_{2}\right.$ and $\left.\mathrm{K}_{2}\right)$ propagate from the southwest as a Kelvin wave and undergo a marked amplification of amplitudes (more than 2.5 times the outer shelf values for all components) after traversing the SMG mouth (Fig. 2, left panels). In the gulf's interior the phase and amplitude changes are small
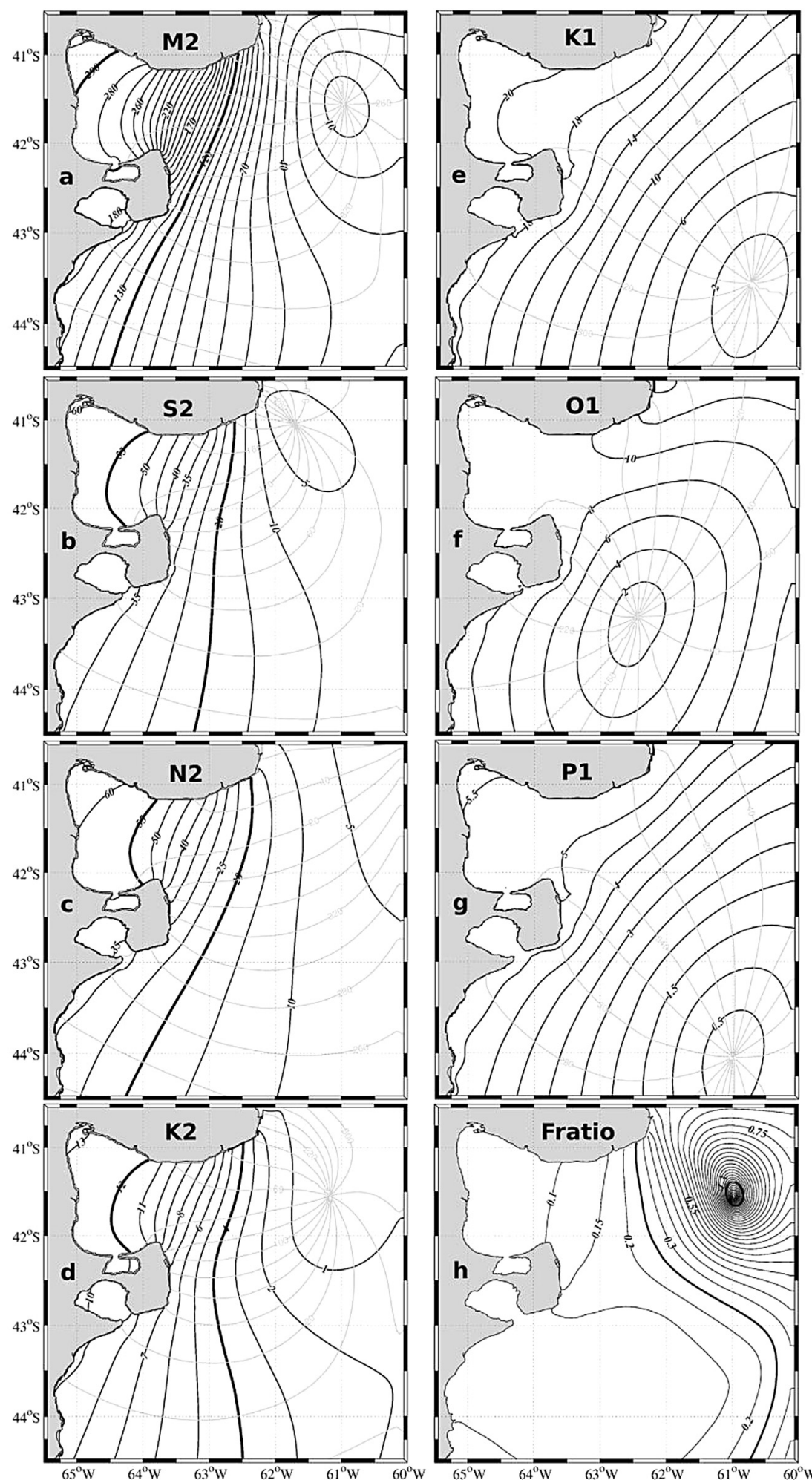

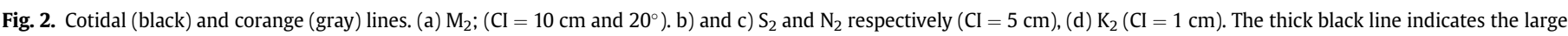
amplification of the semidiurnal waves after transversing SMG mouth. e) and f) $\mathrm{K}_{1}$ and $\mathrm{O}_{1}$ respectively $\left(\mathrm{CI}=2 \mathrm{~cm}\right.$ and $\left.\left.20^{\circ}\right), \mathrm{f}\right) \mathrm{P}_{1}(\mathrm{CI}=0.5 \mathrm{~cm})$, h) Factor amplitude ratio. 
reaching a maximum of $3.14 \mathrm{~m}$ for $\mathrm{M}_{2}$ in the northern coast (San Antonio Bay). The tidal amplification is very weak inside the NG and SJG but the maximum amplitude is substantially larger at SJG $(\sim 3.00 \mathrm{~m})$ than in NG $(\sim 1.80 \mathrm{~m})$ (Fig. 2a). Tidal amplitudes of $S_{2}$ and $\mathrm{K}_{2}$ are about $20 \%$ and $5 \%$ compared with $\mathrm{M}_{2}$ (Fig. $2 \mathrm{~b}$ and $\mathrm{d}$ ). An interesting feature of the NPGs is that the $\mathrm{N}_{2}$ tide is of the same order (and with larger amplitudes in some localities, Fig. 2c) than the $S_{2}$ indicating a large variation of consecutive spring-neap tidal cycles. Co-phase charts show that there is an amphidromic point in the shelf near the northeast corner of the computational domain. In the case of $\mathrm{M}_{2}$ the amphidrome is located at $41^{\circ} 30^{\prime} \mathrm{S}, 61^{\circ} \mathrm{W}$ while for $S_{2}$ is displaced towards the northwest. There is no $\mathrm{N}_{2}$ amphidrome inside the computational domain. For $\mathrm{K}_{2}$ the amphidrome is slightly westward of the $\mathrm{M}_{2}$ location. These differences in the location of amphidromes in a tidal frequency band (i.e. semidiurnal), can be attributed to the differences in frequency and hence wavelength, among constituents in that band (Nye et al., 1988).

Unlike the semidiurnal tides, the diurnal constituents $\mathrm{K}_{1}, \mathrm{O}_{1}$ and $\mathrm{P}_{1}$ do not show any sign of large amplification inside the gulfs. Gulfscale resonance for these constituents is not expected because their propagation speeds are about half those of the semidiurnal constituents. Amplitude maxima are located near the coastal region and in the interior of SMG $\left(\sim 20 \mathrm{~cm}\right.$ for $K_{1}, 8 \mathrm{~cm}$ for $\mathrm{O}_{1}$ and $5 \mathrm{~cm}$ for $\mathrm{P}_{1}$ ) with some amplification in the northern shelf for $\mathrm{O} 1$ (Fig. 2e, $\mathrm{f}$ and $\mathrm{g}$ ). The $\mathrm{Q}_{1}$ tide has maximum amplitudes less than $2 \mathrm{~cm}$ in most of the domain (not shown). For the diurnal components there is one amphidromic point located in the middle shelf southeast of Valdés Peninsula (VP hereafter). For $K_{1}$ the amphidrome is located at $43^{\circ}$ $35^{\prime} \mathrm{S}$ and $60^{\circ} 50^{\prime} \mathrm{W}$, for $\mathrm{O} 1$ it is displaced towards the northwest (inshore) and for $\mathrm{P}_{1}$ it is located slightly southward compared with $\mathrm{K}_{1}$.

The character of the tide in a particular region can be generally described in terms of the amplitude ratio between diurnal and semidiurnal tidal constituents, i.e., $\mathrm{F}=\left(\mathrm{K}_{1}+\mathrm{O}_{1}+\mathrm{P}_{1}\right) /$ $\left(\mathrm{M}_{2}+\mathrm{N}_{2}+\mathrm{S}_{2}+\mathrm{K}_{2}\right)$ (Pugh, 1987). Based on this ratio, the tidal regime (Fig. $2 \mathrm{~h}$ ) of the region can be generally characterized as mainly semidiurnal $(\mathrm{F}<0.25)$. Some contribution is made by the diurnal constituents in the northeast shelf sector where the amplitude minimum of the semidiurnals is located $(0.25<\mathrm{F}<3)$.

Although a rigorous statistical analysis of the model performance is precluded by the scarcity of long-term measurements (a record length larger than 30 days is needed to separate $N_{2}$ from $\mathrm{M}_{2}$ ), our regional model displays a very good correspondence with available measured elevation and phases for almost all constituents (Table 1). The average difference is less than $2.5 \mathrm{~cm}$ in amplitude and $9^{\circ}$ in phase. The amplitude error is larger for the $\mathrm{N}_{2}$ component at Pta. Colorada and San Román (15 $\mathrm{cm}$ and $8 \mathrm{~cm}$ respectively) while the phase discrepancies are larger at Rawson $\left(\sim 15^{\circ}\right)$ for all semidiurnals (except $\mathrm{K}_{2}$ ) and grow a little for the $\mathrm{O}_{1}$ diurnal inside SMG (maximum difference of $36^{\circ}$ at Pta. Colorada). The overall agreement indicates that the primary physical processes affecting the tides in the NPGs are adequately described by forcing the regional model with data extracted from a global tidal model at the open boundary, without assimilation or tuning of the model parameters (bottom friction and/or horizontal viscosity). The present solutions reveal similarities with previous published cotidal charts and also additional features of the NPGs circulation poorly delineated or not reported in earlier low resolution models.

The cotidal charts shows one amphidromic point for the semidiurnal tides off the mouth of SMG and one for the diurnals southeast of VP. This $\mathrm{M}_{2}$ amphidrome have been reported in previous studies but its reproduction relied on some tuning of the bottom friction parameter (i.e., Glorioso and Flather, 1997;
Simionato et al., 2004). Our simulation locates the $\mathrm{M}_{2}$ amphidrome very close to the one reported in model B of Moreira et al. (2011). For the remaining semidiurnal waves $\left(S_{2}, N_{2}, K_{2}\right)$ the model shows displacements of the amphidrome as compared with $\mathbf{M}_{2}$. Glorioso and Flather (1997) also noted the $\mathrm{S}_{2}$ amphidrome displacement towards the coast as compared with $\mathrm{M}_{2}$ but due to the model resolution, it is very difficult to make a more quantitative comparison with their published charts. Additionally, the present high-resolution simulation showed that no new amphidromes are developed inside the Gulfs. The model shows a large amplification of the $\mathrm{M}_{2}$ tide after traversing the SMG mouth, a result already reported in previous models (i.e, Glorioso and Flather, 1997; Moreira et al., 2011). Our results show that this amplification extends to all semidiurnal components, supporting the hypothesis of resonance for the SMG at the semidiurnal frequency. The spatial structure of the diurnal tidal wave has only been explored by Glorioso and Flather (1997). There is a general concordance with the position of the offshore $\mathrm{K}_{1}$ and $\mathrm{O}_{1}$ amphidromes (ours is a little inshore in both cases), but there is no detail in their work about the character of the diurnal waves inside the NPGs as in our model (Fig. 2e and f).

\subsection{Tidal currents}

We now examine the tidal currents mapping the depth-average current ellipses for each constituent (Fig. 3). The semidiurnal currents amplify from the southern shelf to the largest values off the coastal region surrounding VP with maximum intensity approaching $2.5 \mathrm{~m} / \mathrm{s}\left(\mathrm{M}_{2}\right)$ at the northeast and SMG mouth (southern sector) (Fig. 3a). The presence of strong currents is also clear at the mouth of NG (particularly its western sector, $\sim 1.8 \mathrm{~m} / \mathrm{s}$ for $\mathrm{M}_{2}$ ), SJG (eastern sector, $\sim 1.2 \mathrm{~m} / \mathrm{s}$ ) and in San Antonio Bay $(\sim 0.8 \mathrm{~m} / \mathrm{s})$ were the restriction imposed by the narrow entrances forces an increase in speed (Fig. 3e). Inside the Gulfs the current intensity decreases but they are still of the order of $0.2 \mathrm{~m} / \mathrm{s}$ for $\mathrm{M}_{2}$. The ellipses present large eccentricity in the southern shelf being more circular off the mouth of NG. After passing the southern tip of VP the flow acquired a rectilinear pattern and changes the sense of rotation as it approaches the SMG mouth. Inside the gulfs there is an important cross-isobath flow in the coastal region (Fig. 3e). The currents are also very intense for $S_{2}$ and $\mathrm{N}_{2}(\sim 0.5 \mathrm{~m} / \mathrm{s}$, or $25 \%$ of the $\mathrm{M}_{2}$ intensity) and much weaker for $\mathrm{K}_{2}\left(\sim 0.1 \mathrm{~m} / \mathrm{s}\right.$, or $5 \%$ of $\left.\mathrm{M}_{2}\right)$ (Fig. 3 , left panels).

For the diurnal constituents the currents are also intense surrounding VP and comparable with $\mathrm{K}_{2}$, but much weaker in magnitude compared with $\mathrm{M}_{2}, \mathrm{~S}_{2}$ and $\mathrm{N}_{2}$ (maximum of $\sim 0.15 \mathrm{~m} / \mathrm{s}$ for $\mathrm{K}_{1}, 0.10 \mathrm{~m} / \mathrm{s}$ for $\mathrm{O}_{1}$, and $0.04 \mathrm{~m} / \mathrm{s}$ for $\mathrm{P}_{1}$; Fig. 3, right panels). At difference with the semidiurnals however, the maximum amplification of the diurnal tidal currents is not located at the entrance of SMG but towards the outer shelf where $\mathrm{O}_{1}$ reaches a maximum of $0.1 \mathrm{~m} / \mathrm{s}$. Eccentricities in the outer shelf agree with the tendencies from inviscid theory in that the ellipses should be more circular for the diurnal than for the semidiurnal species. This effect is larger for $\mathrm{O}_{1}$ than for $\mathrm{K}_{1}$ and $\mathrm{P}_{1}$ (Fig. $3 \mathrm{~g}$ ). The intensification of the diurnal currents in the outer shelf has been ascribed to resonance between the diurnal wave and the local shelf wave, the period of which is determined by the local cross-shelf topography. (Han, 2000; Palma et al., 2004).

The modeled ellipses are compared with current meter data in Table 2 . The few available observations and the record length limit the comparison to a small number of constituents and preclude a more rigorous analysis. Furthermore, taking into account possible errors due to instrumental accuracy and noise associated with the harmonic analysis of current meter data only magnitudes exceeding $2 \mathrm{~cm} / \mathrm{s}$ are considered in the comparison (Pugh and 
Table 1

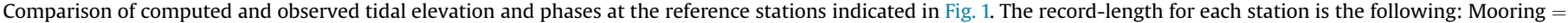
660 days, Pto. Madryn = 365 days, S.A.E., Pta. Colorada and San Román $=31$ days, Rawson $=37$ days.

\begin{tabular}{|c|c|c|c|c|c|c|}
\hline \multirow[t]{2}{*}{ Station } & \multicolumn{2}{|c|}{ Amplitude [meters] } & \multirow[t]{2}{*}{$\Delta \mathrm{h}$} & \multicolumn{2}{|c|}{ Phase [degrees] } & \multirow[t]{2}{*}{$\Delta \varphi$} \\
\hline & Observed & Modeled & & Observed & Modeled & \\
\hline \multicolumn{7}{|l|}{ M2 } \\
\hline Mooring & 2.57 & 2.61 & 0.04 & 71 & 73 & 2 \\
\hline S.A.E. & 3.14 & 3.14 & 0 & 72 & 70 & -2 \\
\hline Pta. Colorada & 2.84 & 2.85 & 0.01 & 63 & 63 & 0 \\
\hline San Román & 2.89 & 2.95 & 0.06 & 71.5 & 62 & -9.5 \\
\hline P. Madryn & 1.88 & 1.85 & -0.03 & 320 & 318 & -2 \\
\hline Rawson & 1.69 & 1.65 & -0.04 & 305 & 290 & -15 \\
\hline \multicolumn{7}{|l|}{ S2 } \\
\hline Mooring & 0.49 & 0.51 & 0.02 & 145 & 154 & 9 \\
\hline S.A.E. & 0.65 & 0.63 & -0.02 & 147 & 153 & 6 \\
\hline Pta. Colorada & 0.56 & 0.57 & 0.01 & 142 & 145 & 3 \\
\hline San Román & 0.6 & 0.59 & -0.01 & 150 & 144 & -6 \\
\hline P. Madryn & 0.45 & 0.44 & -0.01 & 34 & 38 & 4 \\
\hline Rawson & 0.41 & 0.4 & -0.01 & 21 & 7 & -14 \\
\hline \multicolumn{7}{|l|}{ N2 } \\
\hline Mooring & 0.59 & 0.55 & -0.04 & 31 & 34 & 3 \\
\hline S.A.E. & 0.66 & 0.66 & 0 & 30 & 32 & 2 \\
\hline Pta. Colorada & 0.74 & 0.59 & -0.15 & 32 & 25 & -7 \\
\hline San Román & 0.69 & 0.61 & -0.08 & 35 & 24 & -11 \\
\hline P. Madryn & 0.4 & 0.36 & -0.04 & 283 & 281 & -2 \\
\hline Rawson & 0.33 & 0.32 & -0.01 & 263 & 248 & -15 \\
\hline \multicolumn{7}{|l|}{ K2 } \\
\hline Mooring & 0.14 & 0.11 & -0.03 & 145 & 157 & 12 \\
\hline P. Madryn & 0.12 & 0.1 & -0.02 & 33 & 35 & 2 \\
\hline \multicolumn{7}{|l|}{ K1 } \\
\hline Mooring & 0.21 & 0.2 & -0.01 & 6 & 12 & 6 \\
\hline S.A.E. & 0.2 & 0.21 & 0.01 & 7 & 12 & 5 \\
\hline Pta. Colorada & 0.21 & 0.2 & -0.01 & 1 & 9 & 8 \\
\hline San Román & 0.16 & 0.2 & 0.04 & 19 & 8 & -11 \\
\hline P. Madryn & 0.18 & 0.19 & 0.01 & 310 & 318 & 8 \\
\hline Rawson & 0.18 & 0.19 & 0.01 & 300 & 301 & 1 \\
\hline \multicolumn{7}{|l|}{01} \\
\hline Mooring & 0.08 & 0.09 & 0.01 & 302 & 306 & 4 \\
\hline S.A.E. & 0.1 & 0.1 & 0 & 292 & 306 & 14 \\
\hline Pta. Colorada & 0.05 & 0.09 & 0.04 & 267 & 303 & 36 \\
\hline San Román & 0.09 & 0.09 & 0 & 287 & 302 & 15 \\
\hline P. Madryn & 0.07 & 0.09 & 0.02 & 221 & 230 & 9 \\
\hline Rawson & 0.08 & 0.11 & 0.03 & 209 & 209 & 0 \\
\hline \multicolumn{7}{|l|}{ P1 } \\
\hline Mooring & 0.06 & 0.05 & -0.01 & 350 & 347 & -3 \\
\hline P. Madryn & 0.05 & 0.05 & 0 & 294 & 295 & 1 \\
\hline \multicolumn{7}{|l|}{ M4 } \\
\hline Mooring & 0.03 & 0.04 & 0.01 & 75 & 90 & 15 \\
\hline P. Madryn & 0.18 & 0.18 & 0 & 161 & 182 & 21 \\
\hline \multicolumn{7}{|l|}{ MS4 } \\
\hline Mooring & 0.01 & 0.02 & 0.01 & 144 & 162 & 18 \\
\hline P. Madryn & 0.08 & 0.08 & 0 & 243 & 294 & 51 \\
\hline \multicolumn{7}{|l|}{ MN4 } \\
\hline Mooring & 0.015 & 0.02 & 0.005 & 31 & 37 & 6 \\
\hline P. Madryn & 0.09 & 0.1 & 0.01 & 123 & 184 & 61 \\
\hline
\end{tabular}

Vassie, 1976). With these caveats in mind, qualitatively, the comparisons are very good for the semidiurnal constituents with ellipse semimajor amplitudes, eccentricities, and orientations all in agreement. Maximum differences are $8.6 \mathrm{~cm} / \mathrm{s}$ (14\%) in magnitude (station SAE for $\mathrm{M}_{2}$ ), 0.2 in eccentricity (station $\mathrm{R}_{2}$ for $\mathrm{O}_{1}$ ) and $27^{\circ}$ in orientation (station $\mathrm{R}_{2}$ for $\mathrm{N}_{2}$ ).

The modeled semidiurnal current patterns, with maximum currents and low eccentricity surrounding VP and in the mouths of NG and SJG and weaker currents inside the Gulfs, are in general correspondence with earlier modelling efforts but with the exception of Simionato et al. (2004) and Moreira et al. (2011) for the $\mathrm{M}_{2}$ tide, no quantitative comparison with measured values was given. Simionato et al. (2004) analysed two stations, one off NG and the other in the mouth of SMG. An inspection of their plotted ellipses indicates an almost rectilinear pattern with a zonal component in excess of $0.8 \mathrm{~m} / \mathrm{s}$ in SMG and a larger eccentricity with velocity magnitude close to $0.3 \mathrm{~m} / \mathrm{s}$ outside NG in agreement with our results (Fig.3a). Moreira et al. (2011) compare their numerical results for $\mathrm{M}_{2}$ with measurements at four stations in the NPGs area (stations PB, PC, LG and SAE in Table 2). They reported good model performance (amplitude error less than $20 \%$ ), with the exception of one station inside SMG, although no numerical values were provided to make a more rigorous comparison with our results.

\subsection{Tidal energy and dissipation}

Tidal energy flux vectors were computed from the model results using the expression of Pugh (1987):

$\vec{F}_{E}=0.5\left[\rho g D \eta_{o} \vec{U}_{o} \cos \left(\varphi_{\eta_{o}}-\vec{\varphi}_{U_{o}}\right)\right]$ 


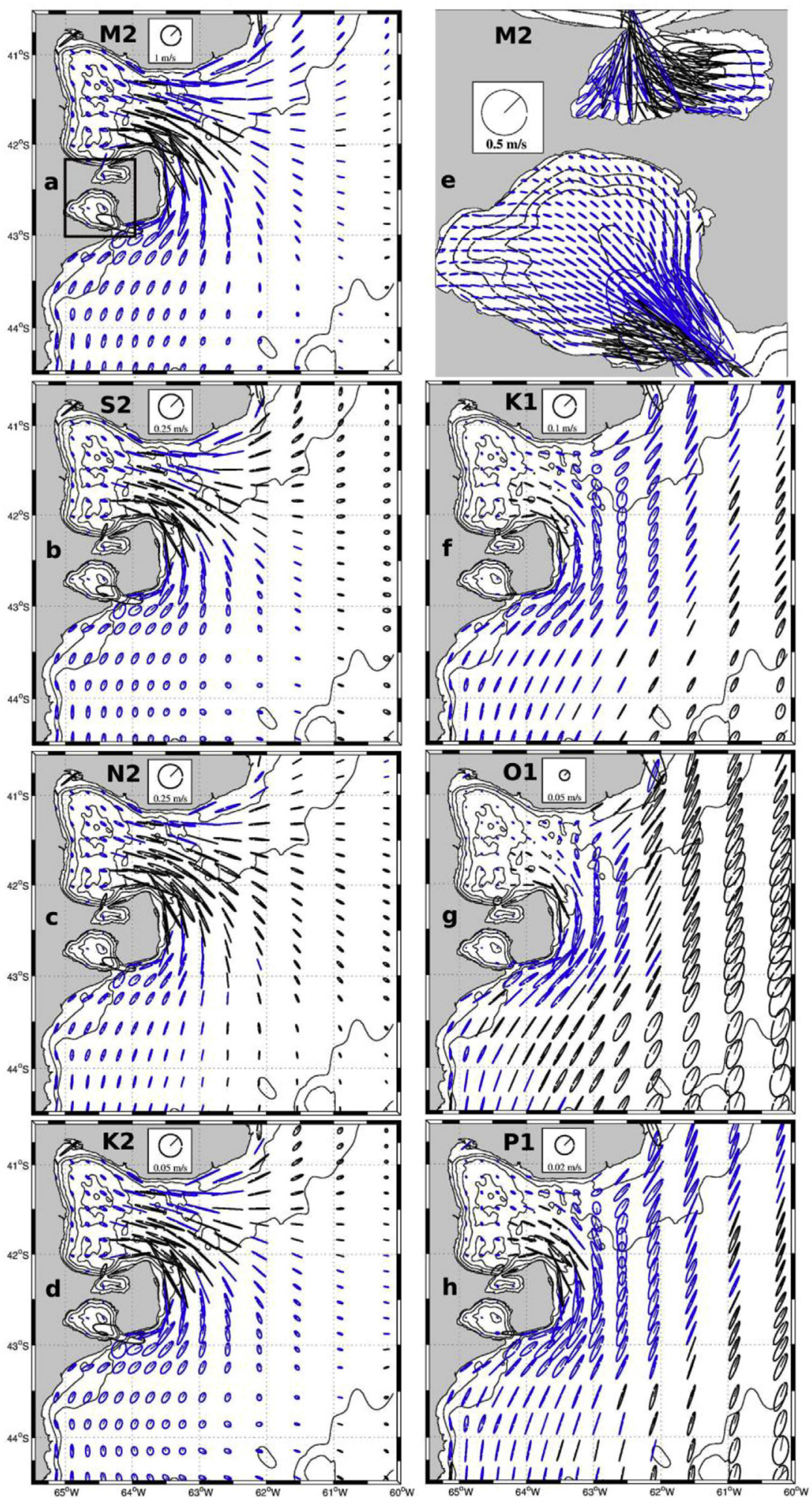

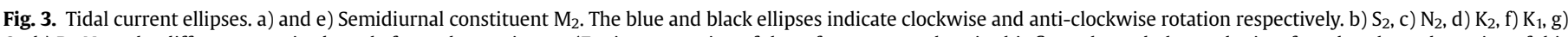

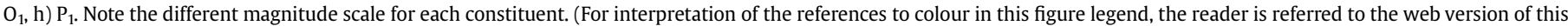
article.)

where $\mathrm{g}$ is gravity, $\rho$ is the seawater density $(1026 \mathrm{~kg} / \mathrm{m} 3), \mathrm{D}$ is the water depth, $\eta_{o}$ and $\vec{U}_{o}$ and $\varphi_{\eta_{o}}$ and $\vec{\varphi}_{U_{o}}$ the amplitudes and phases of the sea surface elevation and velocity components respectively. To identify the areas with the highest tidal energy losses, we 
Table 2

Comparison of computed and observed tidal ellipse parameters at the reference stations indicated in Fig. 1. SEMAX: semi-major axis, ECC: eccentricity, negative values indicate that the ellipse is traversed in a clokwise rotation, INC: angle between East and semi-major axis. The record-length for each station is the following: $\mathrm{R} 1=149$ days, $\mathrm{R} 2=315$ days, $\mathrm{LG}=130$ days, $\mathrm{PB}=35$ days, $\mathrm{PC}=58$ days and S.A.E. $=$ 25 days.

\begin{tabular}{|c|c|c|c|c|c|c|c|}
\hline & \multirow[t]{2}{*}{ Station } & \multicolumn{2}{|c|}{ SEMAX $[\mathrm{cm} / \mathrm{s}]$} & \multicolumn{2}{|l|}{ ECC } & \multicolumn{2}{|c|}{ INC [deg] } \\
\hline & & Obs & Mod & Obs & Mod & Obs & Mod \\
\hline \multirow[t]{6}{*}{ M2 } & $\mathrm{R} 1$ & 43.79 & 42.42 & -0.56 & -0.57 & 320 & 318 \\
\hline & $\mathrm{R} 2$ & 45.92 & 44.15 & -0.14 & -0.26 & 43 & 53 \\
\hline & PB & 4.93 & 8.95 & -0.0061 & -0.024 & 24 & 1 \\
\hline & PC & 18.81 & 14.9 & -0.16 & -0.29 & 108 & 107 \\
\hline & LG & 28.25 & 32.76 & 0.13 & 0.177 & 120 & 115 \\
\hline & S.A.E. & 60.42 & 69.07 & 0.04 & 0.04 & 42 & 33 \\
\hline \multirow[t]{4}{*}{ S2 } & $\mathrm{R} 1$ & 8.52 & 10.26 & -0.53 & -0.41 & 314 & 313 \\
\hline & $\mathrm{R} 2$ & 8.36 & 8.94 & -0.23 & -0.36 & 39 & 51 \\
\hline & LG & 6.36 & 6.92 & 0.05 & 0.15 & 120 & 116 \\
\hline & S.A.E. & 15.83 & 14.6 & -0.13 & 0.05 & 44 & 33 \\
\hline \multirow[t]{3}{*}{ N2 } & $\mathrm{R} 1$ & 8.09 & 7.68 & -0.46 & -0.61 & 308 & 300 \\
\hline & $\mathrm{R} 2$ & 11.28 & 9.51 & -0.08 & -0.19 & 31 & 58 \\
\hline & LG & 6.83 & 6.83 & 0.08 & 0.16 & 121 & 115 \\
\hline \multirow[t]{2}{*}{ K2 } & $\mathrm{R} 2$ & 3.06 & 1.98 & -0.23 & -0.37 & 43 & 48.8 \\
\hline & S.A.E. & 3.23 & 3.2 & -0.18 & 0.05 & 23.6 & 33.7 \\
\hline \multirow[t]{2}{*}{ K1 } & $\mathrm{R} 1$ & 4.17 & 3.9 & -0.57 & -0.56 & 24 & 28.05 \\
\hline & $\mathrm{R} 2$ & 7.23 & 6.32 & -0.05 & -0.09 & 35 & 50.2 \\
\hline \multirow[t]{2}{*}{01} & $\mathrm{R} 1$ & 2.5 & 3.42 & -0.47 & -0.28 & 16 & 29.36 \\
\hline & $\mathrm{R} 2$ & 5.92 & 5.64 & 0.21 & 0 & 39 & 48.03 \\
\hline
\end{tabular}

compute the rate of energy dissipation as:

$D_{E}=\frac{1}{T} \int_{0}^{T} C_{d} \rho\left(u^{2}+v^{2}\right)^{3 / 2} d t$

where $C_{d}$ is the bottom drag coefficient (0.003), $u$ and $v$ are the bottom velocity components, and $\mathrm{T}$ is the tidal period.

The $\mathrm{M}_{2}$ has the strongest currents and highest amplitudes and therefore the largest tidal energy fluxes for the region. The bulk of tidal energy enters the domain from the south $(27 \mathrm{GW})$ and east (3.1 GW) with a small portion leaking through the northern boundary (2.1 GW) (Fig. 4a). As there is convergence of energy fluxes inside the domain, to reach a steady state the energy must be dissipated. The dissipation however is not spatially homogeneous. For a more detailed analysis we subdivide the computational domain in five zones: South Patagonia (SP), North Patagonia (NP), SMG, NG and SJG.

The SP dissipated only $2.8 \mathrm{GW}$ (Fig. $4 \mathrm{a}$ ) of the $29.1 \mathrm{GW}$ that enter from the south and east, and it is concentrated mainly at the southern tip of VP and mouth of NG. The NP receives $27 \mathrm{GW}$ with a small portion of it (10\%) outflowing to the SMG region and northern shelf and dissipating the bulk of the $\mathrm{M}_{2}$ energy (22.4 GW). This is not unexpected, because both strong currents and rough and irregular bottom topography are almost collocated and therefore contribute to the high dissipation rate. The local maxima $(>8 \mathrm{~W} /$ $\mathrm{m}^{2}$ ) are located in two centers around the VP and southern portion of the mouth of SMG (Fig. 4a). After entering SMG the energy flux vectors develop a clockwise gyre and exits through the northern portion of the mouth. Most of the energy is dissipated in the vicinity of the mouth $(2.4 \mathrm{GW})$ and the remaining $(0.1 \mathrm{GW})$ in the entrance of SJG. Very little energy enters the NG and SJG, the bulk being dissipated at the gulfs' entrances.

The distribution of energy flux vectors for the other semidiurnal constituents $\left(\mathrm{S}_{2}, \mathrm{~N}_{2}\right.$ and $\left.\mathrm{K}_{2}\right)$ is not significantly different from that found for $\mathrm{M}_{2}$ (Fig. 4, left panels) although the magnitude is reduced by 50 for $\mathrm{S}_{2}$ and $\mathrm{N}_{2}$ and by 500 for $\mathrm{K}_{2}$. This reduction in energy flux leads to reduced elevations (Fig. 2, left panels), currents (Fig. 3, left

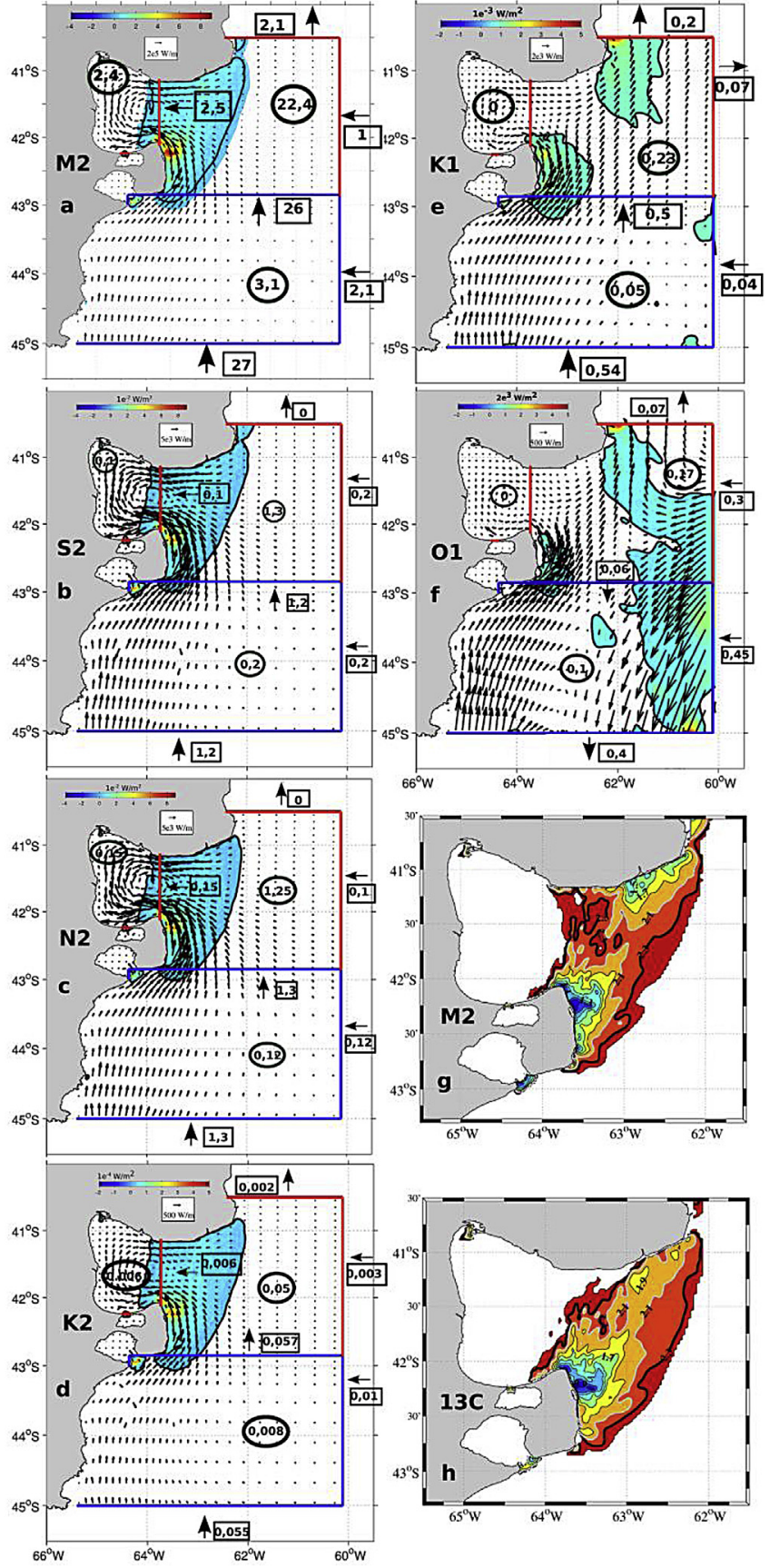

Fig. 4. Tidal energy fluxes (arrows) and dissipation (colours). a) to d) Semidiurnal constituents. (e) and (f) Diurnal constituents. The blue and red lines separate subdomains of analysis: South Patagonian (SP, blue lines), North Patagonian (NP, red lines) and the three gulfs: SMG, NG and SJG. The black circles indicate net energy dissipation into the subdomain. The black box indicates net exchange energy fluxes. g) and h) Simpson - Hunter parameter for the $\mathrm{M}_{2}$ only and the 13 component run respectively. The thick black line is the 2.3 contour. (For interpretation of the references to colour in this figure legend, the reader is referred to the web version of this article.)

panels) and energy dissipation although the spatial patterns are essentially the same as that found for $\mathrm{M}_{2}$. The existence of clockwise gyres of flux vectors for all semidiurnals is indicative of standing waves and resonance at this particular frequency (Glorioso and Flather, 1997).

The $\mathrm{K}_{1}$ energy flux vectors (Fig. 4e) show some similarities but also important differences to those found for the semidiurnals. Although there is energy flux from the southern shelf $(\sim 0.54 \mathrm{GW})$ 
giving rise to the intensification of tidal currents towards the northeast in a similar manner to that found for the semidiurnals (Fig. 3e), after passing VP the energy path differs significantly. A large part of this energy dissipates off VP $(\sim 0.2 \mathrm{GW})$ while the remaining portion veers offshore (Fig. 4e). Therefore little tidal energy enters the SMG at this frequency and there are almost no amplification of the tidal wave and a substantial decrease in tidal currents inside the gulf (Fig. 2e and Fig. 3e). The northeastward energy flow gives rise to a region of intensified tidal currents in the outer northeast shelf (Fig. 3e). The energy flux vectors corresponding to $\mathrm{O}_{1}$ have similar magnitude compared with those of $\mathrm{K}_{1}$ but with important differences in its spatial structure. There are two branches of energy flow for $\mathrm{O}_{1}$. Like $\mathrm{K}_{1}$, the western branch enters the domain from the southern boundary and flows to the northeast. However, very little energy at this frequency surpasses the dissipation center off VP, leading to the observed weakening of elevations and currents inside the SMG (Fig. 3f). More importantly, most of the energy at the $\mathrm{O}_{1}$ frequency enters the domain from the eastern boundary ( $0.7 \mathrm{GW})$ and supports a strong eastern branch of energy flowing southward. This flow is associated with the eastern limb of an anticlyclonic energy flux gyre of $\mathrm{O}_{1}$ centered further south in the deep ocean (Glorioso and Flather, 1997). The energy flux vectors magnitude and dissipation rate for $P_{1}$ and $Q_{1}$ are a small fraction of $K_{1}(<6 \%$, not shown).

Similar spatial patterns of energy dissipation were found in previous shelf (Glorioso and Flather, 1997; Palma et al., 2004; Simionato et al., 2004) and regional models (Glorioso and Simpson, 1994; Moreira et al., 2011) although there are some differences in magnitude. The total area-integrated energy dissipation in our simulations attains $27.9 \mathrm{GW}$, and is in close correspondence with the 28 GW computed by Palma et al., 2004 (including a more extensive shelf) representing $24 \%$ of total energy dissipated in the Southwestern Atlantic Shelf (Palma et al., 2004). The value is higher than the 15 GW computed by Moreira et al. (2011) for a similar region but using a different numerical model ( $z$ coordinate).

Glorioso and Simpson (1994) proposed a critical value S$\mathrm{H}=2.3 \pm 0.2$ of the Simpson and Hunter (1974) parameter to predict the location of mixing fronts in the NPGs based on their analysis of ship and summer satellite images of sea surface temperature data and a numerical $\mathrm{M}_{2}$ tidal model. We computed the $\mathrm{S}$ $\mathrm{H}$ parameter using the $\mathrm{M}_{2}$ currents and also the full 13-component results (Fig. $4 \mathrm{~g}$ and $\mathrm{h}$ ). The spatial regions enclosed by the critical contour $(\mathrm{S}-\mathrm{H}=2.3$ ) are found around and north of VP, the shallow region in the mouth of the gulfs, and in northern SMG. The enclosed area is slightly larger for the $\mathrm{M}_{2}$ run (Fig. $4 \mathrm{~g}$ ) than for the full component run (Fig. 4h) with the critical contour inside the SMG moving offshore in the latter case. The average frontal position obtained from the model is supported by more recent analysis of satellite sea surface temperature showing a pool of low temperature waters to the east and north of VP (Gagliardini and Rivas, 2004; Romero et al., 2006; Pisoni et al., 2014) and are also in good correspondence with the S-H critical contours computed by earlier models (Glorioso and Simpson, 1994; Moreira et al., 2011). The largest (although not substantial) differences were detected when comparing with the low resolution model of Glorioso and Flather (1995). In their simulations the critical contour off VP ends up at lower latitude and due to resolution issues there is no signature of critical contours near the mouths of NG, SJG or San Antonio Bay.

\subsection{Nonlinear tidal waves}

The presence of nonlinear advection terms and quadratic bottom friction in the model equations give rise to higher harmonics of the astronomical tides. Here we used the model results to analyze the $\mathrm{M}_{4}$ overtide and the $\mathrm{MN}_{4}$ and $\mathrm{MS}_{4}$ compound tides.
The cotidal charts for the $\mathrm{M}_{4}$ and $\mathrm{MS}_{4}$ constituents (the results for $\mathrm{MN}_{4}$ are very similar to that of $\mathrm{MS}_{4}$ ) are shown in Fig. 5 (top panels). At difference with $\mathrm{M}_{2}$, the largest amplification of the higher harmonics occurs at the northeast of VP and most notably, inside NG. There is an amphidromic point for $\mathrm{M}_{4}$ located in the outer shelf southeast of VP that is displaced $\sim 30 \mathrm{~km}$ southward for $\mathrm{MS}_{4}$ and $\mathrm{MN}_{4}$. The amplitude error is very small for the higher harmonics at Madryn, the westward end of NG (less than $1 \mathrm{~cm}$ ) but grows for the phases $\left(\sim 20^{\circ}\right.$ for $\mathrm{M}_{4}$ and $60^{\circ}$ for $\mathrm{MN}_{4}$, Table 1$)$. The gradient of the phase field is very large at NG mouth (see inserts in Fig. 5), therefore small changes in the bathymetric features at this location can induce large phase errors inside the gulf. The total tidal amplitude at Madryn adding the three nonlinear tides amounts to $34 \mathrm{~cm}$ that is $20 \%$ of the $\mathrm{M}_{2}$ and of the same order as $\mathrm{S}_{2}$ and $\mathrm{N}_{2}$ at this location (see also Table 1). The $\mathrm{M}_{4}$ and $\mathrm{MS}_{4}$ current ellipses showed similar intensity and distribution patterns as the semidiurnal waves, but the large current amplification is located in the mouth of NG $\left(\sim 0.4 \mathrm{~m} / \mathrm{s}\right.$ for $\mathrm{M}_{4}$ and $\sim 0.15 \mathrm{~m} / \mathrm{s}$ for $\mathrm{MS}_{4}$ and $\mathrm{MN}_{4}$, inserts in Fig. 5b and e). Moreira et al. (2011) have also registered an amplification of the $\mathrm{M}_{4}$ tide inside $\mathrm{NG}$, although their maximum modeled amplitude is lower than the observed $(0.10 \mathrm{~cm})$. We did ancillary experiments (not shown) removing the higher harmonics from the open boundary forcing and found lower amplitudes inside NG. These results show the importance of including the higher harmonics as a boundary forcing to properly represent its dynamics inside the domain (Davies and Lawrence, 1994).

The energy flux for the quarter-diurnal harmonics shows two different paths. There is a northeastward flow of energy coming from the southern boundary that converges towards NG but also energy outflow from the SMG that leaks towards the outer shelf (Fig. $5 c$ ). The energy that enters the NG plus the local generation forms a closed gyre spanning the entire gulf (Fig. 5c, insert). Results from $\mathrm{MS}_{4}$ and $\mathrm{MN}_{4}$ (the later not shown) are very similar although their magnitudes are about one-fourth of the $\mathrm{M}_{4}$ fluxes. Glorioso and Flather (1997) have associated tidal energy gyres at the semidiurnal frequency in the Patagonian Shelf (for example inside SMG) to the presence of standing waves and therefore the possibility of resonance. The amplification of the quarter-diurnal waves inside NG might also be related to a resonance phenomenon at these frequencies. The export of energy at quarter-diurnal frequency from the Patagonian Shelf towards the deep ocean has been documented by altimeter studies and high resolution models of the Atlantic Ocean (Ray, 2007; Pairaud et al., 2008). Glorioso and Flather (1997) showed a generation center of energy at this frequency in the coastal region near $51^{\circ} \mathrm{S}$ that leaks towards the shelf but also towards the SMG. In contrast, our results showed that inside the SMG there is a transfer of energy from the $\mathrm{M}_{2}$ to its higher harmonics and that this energy is funneled to the outer shelf reinforcing the observed leaking into the Atlantic Ocean.

\section{Tidal residual circulation and dynamics}

In macro-tidal regions the time-mean (residual) currents are generally of the same order as the instantaneous currents and therefore dominate over other forcing mechanisms, like winds or buoyancy. The agreement between the modeled elevations and current ellipses with previous models and observed data are reasonable and can provide an acceptable estimation of the residuals in the NPGs. In this section we described the residual circulation patterns produced by the model and examined the physical mechanisms responsible for these patterns.

Fig. 6a shows the Eulerian residual currents and associated stream function from the benchmark experiment (BEN) averaged over the last 30 days of the simulation. The BEN set-up was described in section 2.1 although in this case only the $\mathrm{M}_{2}$ tide is 


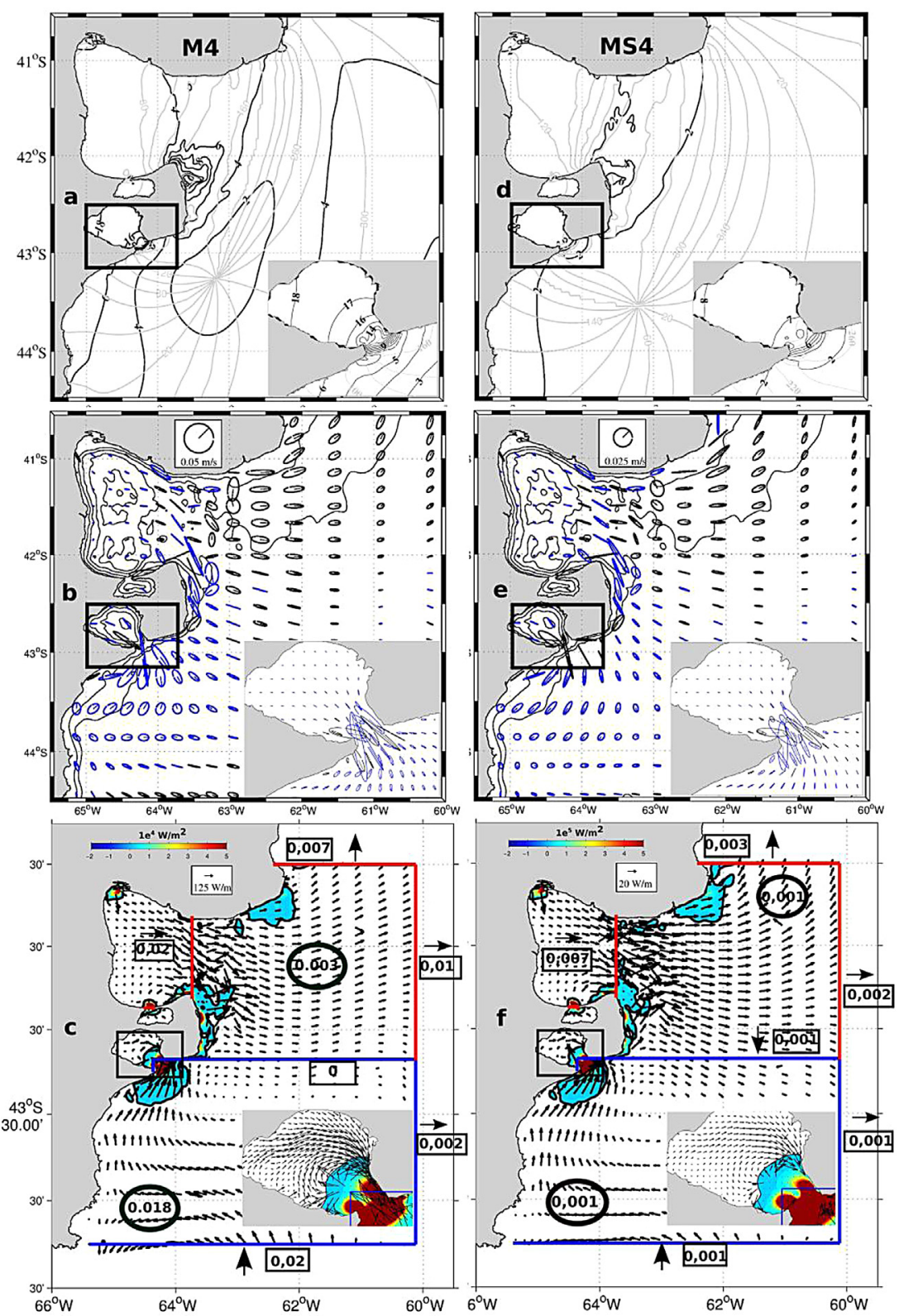

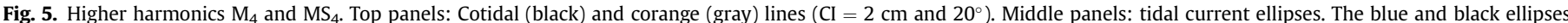

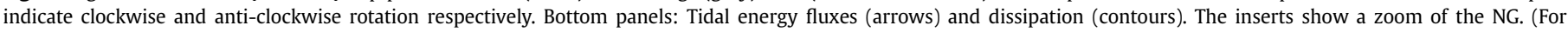
interpretation of the references to colour in this figure legend, the reader is referred to the web version of this article.)

imposed at the open boundary. From south to north there is a coastal current flowing with the coast to the left that intensifies west of VP (Valdés Coastal Current, VCC). The VCC shows two large meanders before leaving the coast near the tip of the peninsula and flowing to the NE. A small branch of the VCC feeds an intense anticyclonic vortex and then rejoins the coast and flows to the east entering SMG. Near the SJG entrance the eastward flowing coastal current intensifies and then turns northward following the SMG shelf topography. As it flows northward the current magnitude diminishes splitting in a number of eastward flowing branches that fills the SMG interior. In the southern portion of SMG the eastward circulation joins a strong cyclonic recirculating gyre of $\sim 0.08 \mathrm{~Sv}$ $\left(1 \mathrm{~Sv}=1 \times 10^{6} \mathrm{~m}^{3} / \mathrm{s}\right)$ centered near the SJG entrance. The northern part of SMG is occupied by a broad southeastward current. The NG shows a gulf-wide cyclonic gyre roughly following the isobaths with intense coastal currents particularly in the southern coast, in agreement with a similar result derived by Rivas (1989). The maximum transport of this gyre is $\sim 0.05 \mathrm{~Sv}$. Superimposed on these regional features there are smaller recirculation patterns. The entrances of NG and SJG for example show pairs of cyclonic/anticyclonic vortexes, which strongly influence the exchange between the SJG and the SMG or between the NG and the shelf. Offshore recirculation patterns associated with banks or depressions are also visible northeast of VP. The largest residual currents $(\sim 30 \mathrm{~cm} / \mathrm{s})$ are found near the mouths of NG and SJG, at the southern coast of NG and SMG and offshore of VP. Computation of the residual currents including the 13 components and averaging the results for 360 days gives similar patterns but the currents' magnitude is weakened (not shown). This is related to an increase in the bottom velocities and therefore the bottom friction brought by the additional components (Marinone, 1997).

Summarizing, the tidal residual circulation in the NPGs can be 


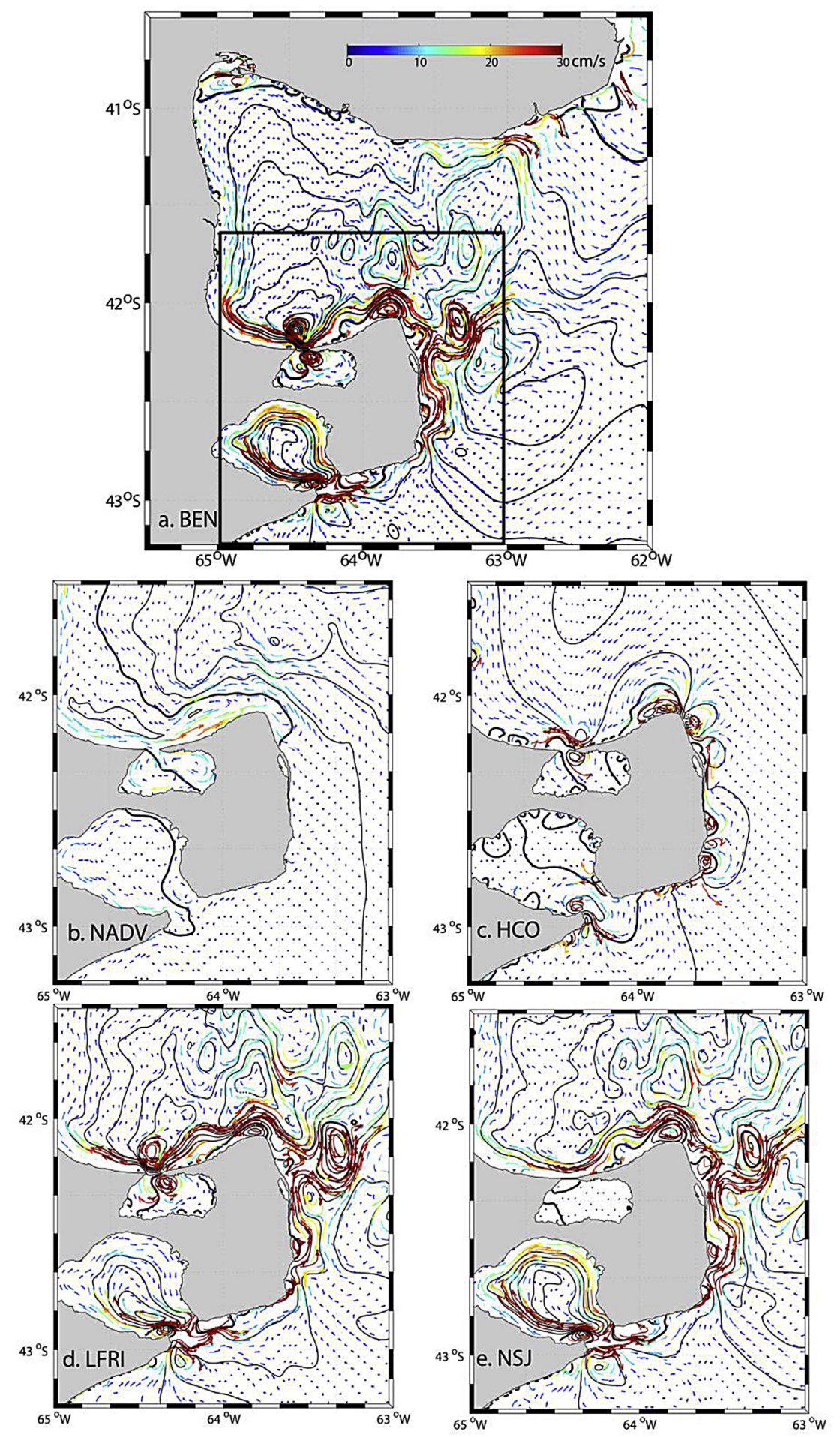

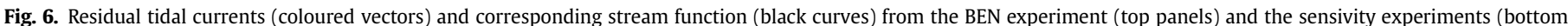
panels). The thick black line is the zero contour of the stream function.

classified in three main patterns: (i) Regional coastal currents and gulf-wide gyres; (ii) Headland, or inlet eddies (Ridderinkhof, 1989) near the mouth of the NG and SJG and (iii) Topographic eddies over small scale topographic features, particularly northeast of VP. Some of these patterns are consistent with previous models although they were less defined due to lower spatial resolution (Glorioso and Simpson, 1994). Unfortunately there are only very short time series of current measurements in the region and therefore there is no direct observational evidence to confirm the modeled patterns or the magnitude of the residual currents. In the following the robustness of the circulation structures will be analysed using vorticity balances, additional sensitivity experiments and comparison with idealized models (i.e. Park and Wang, 1994). The sensitivity experiments are: (i) experiment neglecting the non-linear advection term in the momentum equations (NADV), (ii) experiment with constant depth (HCO), (iii) experiment with linear 
bottom friction terms (LFRI) and finally (iv) experiment with SJG closed (NSJ).

The vorticity balances are based on the transport vorticity equation derived taken the curl of the model's equations of motion (Park and Wang, 1994):

$$
\begin{array}{ccccccc}
\frac{\partial \Omega}{\partial t}+\operatorname{curl}_{z}(A D V) & -f \frac{\partial \eta}{\partial t}+\beta V D= & -g J(\eta, D) & -\operatorname{curl}_{z}\left(\tau_{b}\right)+\operatorname{curl}_{z}(D I F F) \\
\mathrm{T}_{1} & \mathrm{~T}_{2} & \mathrm{~T}_{3} & \mathrm{~T}_{4} & \mathrm{~T}_{5} & \mathrm{~T}_{6} & \mathrm{~T}_{7}
\end{array}
$$

where $\Omega=\frac{\partial(V D)}{\partial x}-\frac{\partial(U D)}{\partial y}$ is the vorticity of the horizontal volume transport $\mathbf{V}_{\mathrm{t}}=(U D, V D)$ or the transport vorticity, $(U, V)$ are the horizontal components of velocity, $D$ is the water depth, $\eta$ the sea surface elevation, $f$ the Coriolis parameter and $\beta$ its meridional gradient, $J$ is the jacobian of two functions, $\operatorname{curl}_{z}$ is the vertical component of the curl, $\tau_{\mathrm{b}}$ is the bottom stress, ADV indicates the nonlinear advection terms and DIFF the turbulent diffusion terms. The Stretching $\left(T_{3}\right)$, Beta $\left(T_{4}\right)$ and diffusion $\left(T_{7}\right)$ terms are negligible compared with the other terms in our simulation and will be omitted in the analysis. The physical mechanisms of vorticity production and dissipation are best evidenced by expanding the bottom friction term $\left(\mathrm{T}_{6}\right)$. Assuming a non-linear (quadratic) bottom friction parameterization of the form:

$\tau_{\mathbf{b}}=\frac{C_{d}\left\|\mathbf{V}_{t}\right\| \mathbf{V}_{t}}{D^{2}}=\frac{\lambda_{t}}{D^{2}} \mathbf{V}_{t}$

where $\left\|\mathbf{V}_{\mathrm{t}}\right\|$ is the transport magnitude, $\lambda_{\mathrm{t}}=\mathrm{C}_{\mathrm{d}}\left\|\mathbf{V}_{\mathrm{t}}\right\|$, and $\mathrm{C}_{\mathrm{d}}$ is a nondimensional bulk friction coefficient we get:

$$
\begin{array}{cccccc}
\frac{\partial \Omega}{\partial t}+\operatorname{curl}_{z}(A D V)=-g J(\eta, D) & -\frac{2 \lambda_{t}\left(\mathbf{V}_{t}\right)}{D^{3}} \times \nabla D+\left(\mathbf{V}_{t}\right) \times \frac{\nabla \lambda_{t}}{D^{2}}-\frac{\lambda_{t}}{D^{2}} \Omega \\
\mathrm{T}_{1} & \mathrm{~T}_{2} & \mathrm{~T}_{5} & \mathrm{~T}_{8} & \mathrm{~T}_{9} & \mathrm{~T}_{10}
\end{array}
$$

Therefore, the vorticity balance indicates that the transient transport vorticity $\left(T_{1}\right)$ is induced from the nonlinear advection $\left(T_{2}\right)$ of the instantaneous vorticity produced when the tidal flow crosses the isobaths, (topographic vorticity tendency, $T_{5}$ ) and differential friction over an irregular coastline or sea bed (terms $\mathrm{T}_{8}$ and $\mathrm{T}_{9}$ ) (Robinson, 1983). Term $\mathrm{T}_{5}$ is also called bottom-pressure torque or just bottom torque (Klinck, 1991). For definiteness we will adopt this last denomination. The last term $\left(\mathrm{T}_{10}\right)$ represents the irreversible dissipation of vorticity by bottom friction. The production terms $T_{5}$ and $T_{8}$ arises in the presence of variable bottom topography (variations normal to the local flow direction in $T_{8}$ ) with $T_{8}$ different from zero even with linear friction formulations. $T_{9}$ depends on the gradient of the mean flow and can exist with constant topography (it is zero for linear friction formulations). In the following we will call $\mathrm{T}_{8}$ vorticity generation by topographic friction, $T_{9}$ vorticity generation by shear friction and $T_{10}$ vorticity dissipation. Taking the temporal average of Eq. (5) over several tidal cycles, we obtain the residual vorticity equation:

$$
\begin{aligned}
& \overline{\operatorname{curl}_{z}(A D V)}=-g \overline{J(\eta, D)}-\overline{\frac{2 \lambda_{t}\left(\mathbf{V}_{t}\right)}{D^{3}} \times \nabla D}+\overline{\left(\mathbf{V}_{t}\right) \times \frac{\nabla \lambda_{t}}{D^{2}}}-\overline{\frac{\lambda_{t}}{D^{2}} \Omega} \\
& \begin{array}{lllll}
R_{2} & R_{5} & R_{8} & R_{9} & R_{10}
\end{array}
\end{aligned}
$$

where the overbar indicates the time average. $R_{2}$ is called residual advection, $R_{5}$ residual bottom torque, $R_{8}$ residual topographic friction, $R_{9}$ residual shear friction and $R_{10}$ residual dissipation.

The paramount importance of the non-linear advection of vorticity $\left(R_{2}\right)$ for the establishment of the residual patterns is illustrated with the results of an experiment where the non-linear advection terms in the equation of motion were neglected (NADV). In this case, all of the residual patterns of BEN, particularly the topographic and inlet eddies vanished (Fig. 6b). Furthermore, the gulf-wide circulation in NG and west of SMG weakens considerably and even change sign. Below we employed vorticity balances to identify the dominating physical mechanisms that account for the main residual patterns.

\subsection{Regional coastal currents and gulf-wide gyres}

Our hypothesis is that the intense VCC and the coastal current at the southern and western coasts of SMG as well as the basin-wide gyre in NG are generated through a tidal rectification mechanism very similar to the one present in straight shelf-slope regions of the open ocean (Pérenne et al., 2000). In terms of the vorticity balance, during the flood tide the water columns cross the isobaths and generate positive vorticity through the bottom torque mechanism $\left(T_{5}\right)$. During the ebb tide there is generation of negative vorticity at the base of the bottom slope. On average there is a residual of positive vorticity on the inshore side that promotes the development of a cyclonic current via non-linear advection. Fig. 7 shows a close-up of the residual transport vorticity in the study area. In regions where the coastal current do not present large meanders (like the southern coast of SMG), the averaged vorticity is positive on the shallower side (facing the current direction) and negative on the deeper side. In NG the bathymetric contours are closed and therefore the coastal current forms a closed cyclonic gyre (Fig. 6a). The VCC, accompanying changes in the bathymetric contours of the coastal region, shows large meanders and the above vorticity pattern is somehow broken by the appearance of centers of positive and negative vorticity (Fig. 6a and Fig. 7).

Further insight into the physical mechanisms responsible for the generation of the coastal currents can be gained analyzing the

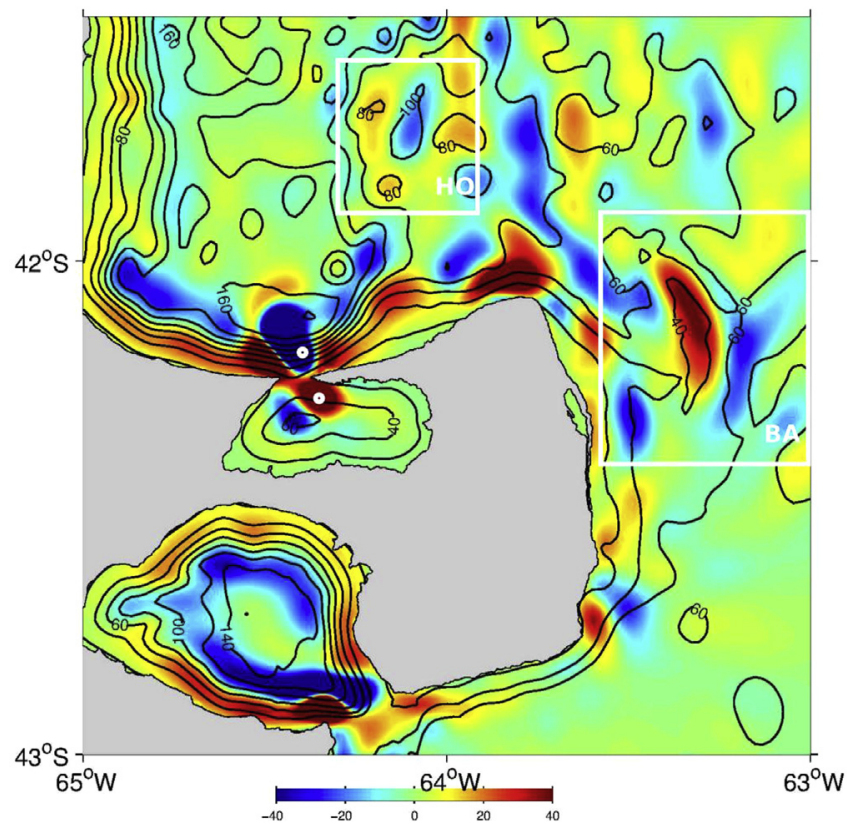

Fig. 7. Residual transport vorticity. Red contours indicates positive (anticyclonic) values and blue contours negative (cyclonic) values. The white circles indicate the stations analysed in Fig. 8. The white rectangles ( $\mathrm{HO}$ and $\mathrm{BA}$ ) indicate the areas analysed in Fig. 9. (For interpretation of the references to colour in this figure legend, the reader is referred to the web version of this article.) 
sensitivity experiments. HCO (Fig. 6c) shows that the most important vorticity production mechanism for the coastal current generation is related to bottom depth variations $\left(\mathrm{T}_{5}\right.$ and $\left.\mathrm{T}_{8}\right)$. Without them the coastal current broadens and weakens considerably and the NG gyre vanishes. The residual circulation in NG and the coastal current, however, is modulated by the production of vorticity by shear friction $\left(\mathrm{T}_{9}\right)$, the currents being more intense and narrow in BEN than in LFRI (where T9 is null) (Fig. 6a and d).

\subsection{Inlet eddies}

The generation of residual eddies around headlands or coastal promontories by strong tidal flows has received a lot of attention in the past (i.e. Pingree, 1978; Ridderinkhof, 1989; Signell and Geyer, 1991; Park and Wang, 2000). It is clear that the residual headland eddies stems from the advection of tidal vorticity generated by the oscillating flow, but the production mechanisms differ. In order to compare these idealized studies with our realistic regional model we computed the transient (Eq. (5)) vorticity balance at two different stations near the SJG entrance's right promontory, where the inlet eddies are well defined (one outside and one inside the gulf, white circles in Fig. 7). In the outside node (north of the mouth) the transport vorticity tendency $\left(\mathrm{T}_{1}\right)$ is dominated by the vorticity advective term $\left(T_{2}\right)$ and the bottom torque $\left(T_{5}\right)$ while the effect of topographic and shear friction is small (Fig. 8a and b). The bottom torque is mostly positive during the flood (flow into SJG) and negative during the ebb (flow out). The vorticity advective term is mostly negative and out of phase with the bottom torque (Fig. 8a). There is a larger efflux of negative vorticity during the ebb than the influx of positive vorticity during the flood. The situation is reversed for the inside node (Fig. 8d), with the advective term mostly positive, although in this case there is a comparable contribution of bottom torque and shear friction during the tidal cycle (Fig. 8d and e).

On average, the residual vorticity is negative (cyclonic) at the north side and positive (anticyclonic) at the south-side (Fig. 8c and f). Note that, as the inlet is flanked by two headlands a similar vortex-pair is visible on the western side of the mouth (Fig. 7). This vortex-pair however is of weaker magnitude compared with the one formed in the eastern side (and the sign is inverted).
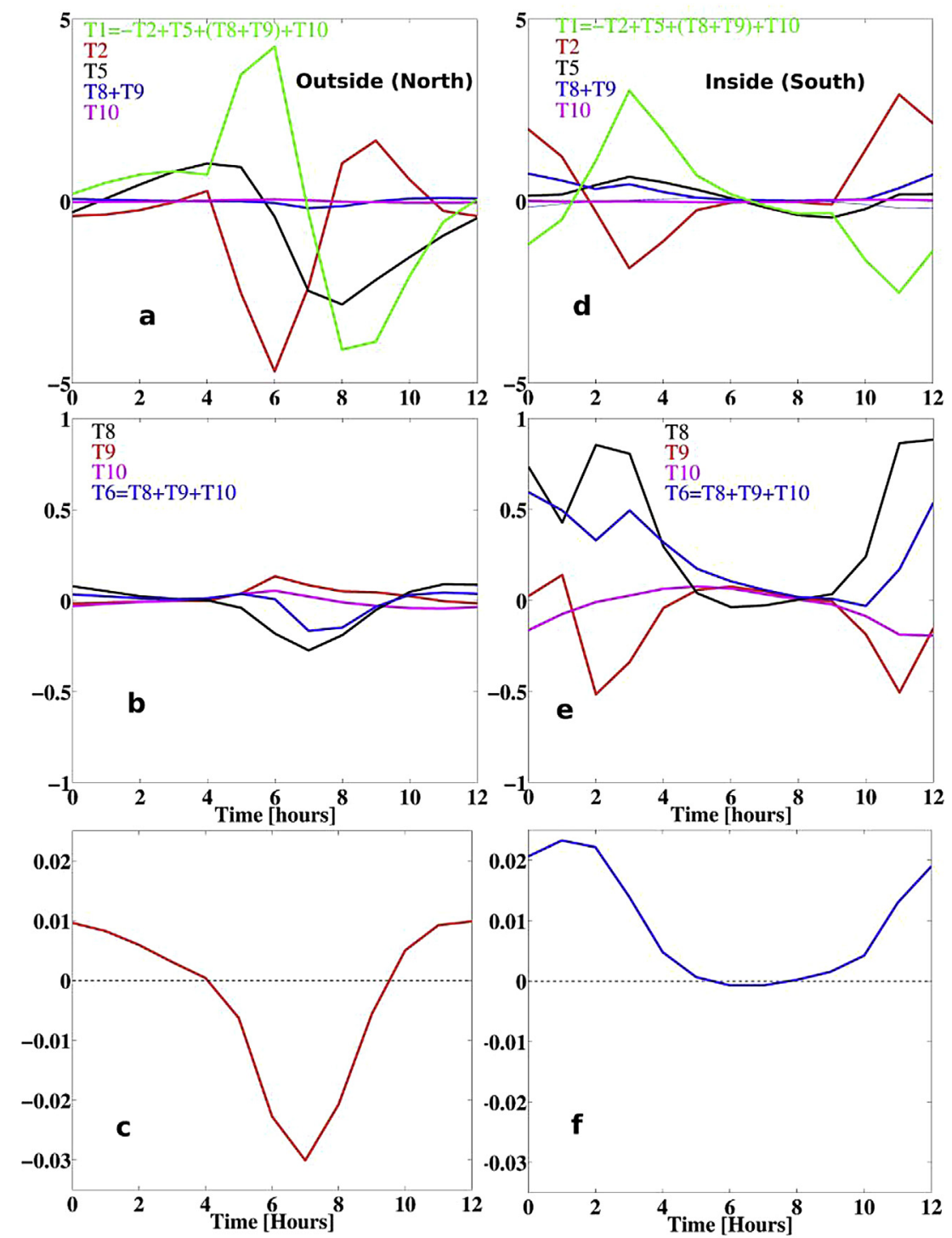

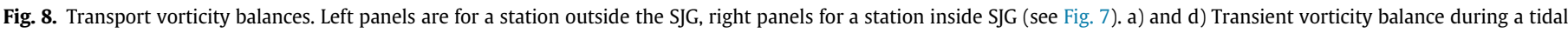
cycle. b) and e) Transient vorticity frictional terms. (c) and (f) Transport vorticity during a tidal cycle. 
Ridderinkhof (1989) have described and analysed a similar "quadrupole" structure near an inlet of the Western Dutch Wadden Sea. An analogous eddy structure is also observable near the NG inlet (Fig. 7).

Park and Wang (2000) in their experiments employed a Gaussian promontory without coastline indentation and a small bottom slope. The appearance of the vortex pair around the promontories in our simulations however does not need the bottom slope. HCO, with a realistic coastline but constant bottom topography showed that the only residual patterns that remain in this case are the vortex pairs near the gulfs' inlets or in regions with strong changes in coastline orientation, like the northeast tip of VP (Fig. 6c). Signell and Geyer (1991) indicate the necessity of a boundary layer separation (and therefore a non-slip condition on land boundaries) to produce the residual headland eddies. Pingree and Maddock (1980) on the other side showed that lateral boundary layers need not necessarily be present to simulate headland eddies. In their model a headland eddy stems from the advection of vorticity generated by differential bottom friction $\left(\mathrm{T}_{8}\right.$ plus $\mathrm{T}_{9}$ ). HCO (with a slip condition on lateral boundaries) shows that part of the vorticity production of the inlet eddies comes indeed from shear friction ( $\left.\mathrm{T}_{9}\right)$, although in BEN topographic friction $\left(\mathrm{T}_{8}\right)$ seems more relevant (Fig. $8 \mathrm{~b}$ and e).

It is interesting to note that the strength of the intense cyclonic vortex located outside the SJG inlet seems to be related to additional mechanisms of vorticity production. A close inspection of the transient vorticity balance at the vortex center shows that the vorticity tendency is in phase with the non-linear vorticity advection and with bottom torque playing a secondary role (Fig. 8a). We postulate that the intensity of this vortex is mostly related to vorticity generated at the south side of the promontory and advected towards the SMG. If this mechanism of vorticity advection is prevented (as in experiment NSJ) the cyclonic vortex almost vanished (Fig. 6e).

\subsection{Topographic eddies}

Closed residual patterns over small topographic features like banks or hollows have been studied by Zimmerman (1980) using analytical models and by Park and Wang (1994) in idealized numerical experiments. According to their studies, the eddies are generated by the non-linear advection of vorticity produced by topographic mechanisms $\left(\mathrm{T}_{5}\right.$ and $\left.\mathrm{T}_{8}\right)$. The importance of these mechanisms is clearly illustrated in the results of NADV and HCO. Without non-linearities or bottom topography gradients the small eddies disappear (Fig. $6 \mathrm{~b}$ and c). The results of BEN, however, showed that there are centers of residual positive (negative) vorticity over banks (hollows) (Fig. 6a and Fig. 7).

The relative importance of the vorticity-production mechanisms is further investigated by vorticity balances along selected features (one bank and one hollow, see Fig. 7 and Fig. 9, top panels). The transient transport vorticity close to the top of the bank is dominated by the bottom torque $\left(T_{5}\right)$ although the topographic friction $\left(\mathrm{T}_{8}\right)$ and shear friction $\left(\mathrm{T}_{9}\right)$ are also important, particularly at those instants (ebb and flood) where $T_{5}$ is small and $T_{8}$ is not completely counterbalanced by shear friction $\left(T_{9}\right.$, Fig. $9 b$ and $\left.c\right)$. Note that at difference with differential friction, bottom-torque is mostly harmonic and in phase with the vorticity tendency $\left(\mathrm{T}_{1}\right)$. The balance is closed by vorticity dissipation $\left(\mathrm{T}_{10}\right)$ that is larger during the ebb and flood phases of the tide. The pattern of residual transport vorticity shows large positive vorticity over the top of the bank and two negative vorticity centers at the sides (the center on the left flank is much smaller than the one on the right flank, Fig. 9a). At the top of the bank the strongest eddy is anticyclonic and at the left/right sides eddies are cyclonic and more attenuated and diffuse. In order to understand how the residual transport vorticity arises, we analyze the residual balance on a zonal transect cutting the bank (Fig. 9a and d). The balance is dominated by vorticity advection $\left(R_{2}\right)$ and residual differential friction $\left(R_{8}+R_{9}\right)$ and in less extent by the residual bottom torque $\left(R_{5}\right)$. This result is a consequence of the instantaneous vorticity balance. Although at the center of the bank the bottom torque is the main source of transient vorticity (Fig. 9b) it is largely harmonic and when averaged, it is overcome by differential friction. Of the two frictional mechanisms of vorticity production, topographic friction is the more important. It is mostly positive during the tidal cycle on top of the bank (Fig. 9c) and negative on the sides (not shown). As a result the combined residual of differential friction $\left(R_{8}+R_{9}\right)$ is positive on top of the bank and negative on the sides (Fig. 9d). The vorticity advective term is largely positive during the tidal cycle (Fig. 9b) and therefore the residual vorticity advective term $\left(R_{2}\right)$ has a maximum positive value near the center of the bank (Fig. 9d). On the flanks the residual advective term is smaller and negative. In summary, the residual transport vorticity balance is dominated by the residual vorticity advective term and the residual topographic friction term (Fig. 9d). This result is consistent with the results of the sensitivity experiments. The topographic eddies generated in LFRI (including $\mathrm{T}_{2}$ and $\mathrm{T}_{8}$ but excluding $\mathrm{T}_{9}$ ) are very similar to those of BEN (Fig. $6 \mathrm{~d}$ ).

A similar counter-rotating group of eddies exists over a hollow. As the bottom slope changes sign from bump to hollow, the signs of the transient transport vorticity and the residual vorticity also change. The residual transport vorticity is anticyclonic at the top of a bank, whereas it is cyclonic at the center of a hollow (Fig. 9e). The transient balance shows that the bottom torque is also the major source for the transient vorticity generation over the hollow. At difference with the bank case however, in this case there is a minor contribution from the frictional torques (Fig. 9g). As a consequence, the residual transport vorticity is largely controlled by the residual vorticity advection and bottom torque (Fig. 9h).

Overall, the above results are in reasonable agreement with the idealized experiments of Park and Wang (1994), particularly with respect to the central eddy (the one on top of the topographic feature). They found the formation of a large residual anticyclonic eddy over a bump with cyclonic eddies on the flanks and of a cyclonic eddy over a hollow with much smaller anticyclonic eddies on the sides. Their vorticity analysis showed that the residual vorticity over the bump is mostly controlled by non-linear advection and differential friction while for the hollow the balance is mostly between non-linear advection and bottom-torque. Our realistic model results also show some differences with the idealized study. The side eddies are not so well defined due to differences in the geometric aspect of the topography (i.e., our selected bank is elongated rather than circular), the proximity of other bathymetric features (small banks surrounding the selected hollow) or other mechanisms involved in the local dynamics. Similar results also applied to other small scale topographic features of the region (anticyclonic vorticity over banks, cyclonic vorticity over hollows) although sometimes the closeness of topographic features with different scales or bottom slope generates centers of alternate sign that precludes a detailed comparison with the idealized studies (i.e. Fig. 9e, where five banks of different height surround a larger hollow).

\section{Summary and discussion}

The strong influence of tidal forcing on the dynamics of coastal regions has been analysed in other major macrotidal areas of the ocean including Davies and Kwong (2001) for the NW European Shelf, Chen et al. (2001) for the Gulf of Maine-Bay of Fundy region and Kang et al. (1998) for the Yellow Sea among others. In 

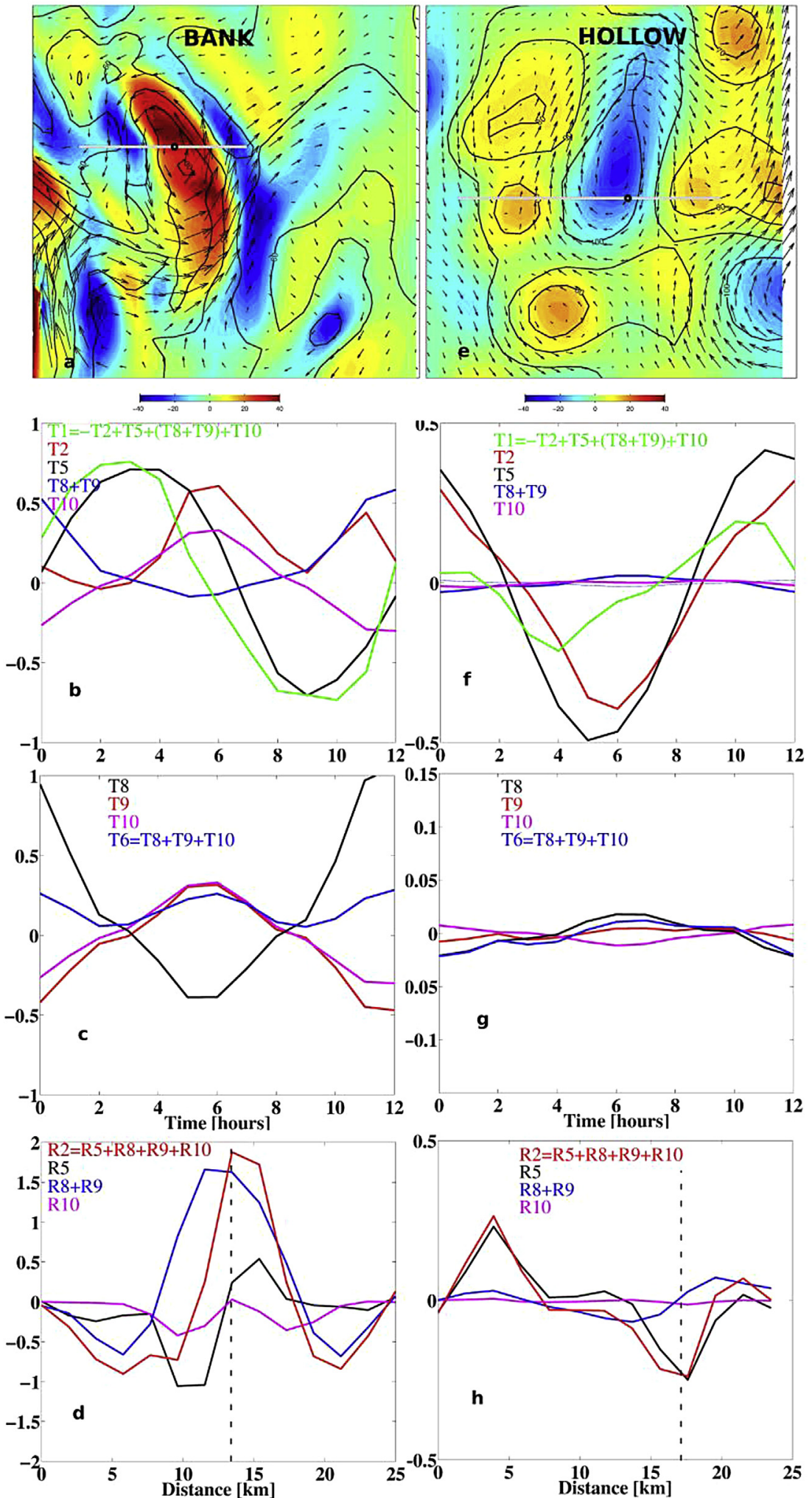

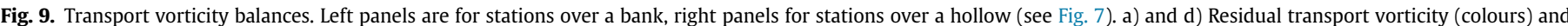

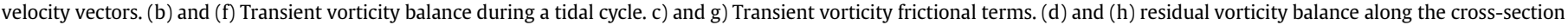
indicated in the top panels. The vertical broken lines in this panels indicate the position of the small circle in the top panels.

comparison there have been relatively few works on the Patagonian Shelf tides, with the model of Glorioso and Flather (1997) setting a baseline for future work. Recent studies however, have shown that the accuracy of previous low-resolution numerical models of the Patagonian Shelf are still unsatisfactory (Saraceno et al., 2010). Much progress has been made on tidal modelling in 
the past decades using assimilation of satellite altimetry (i.e He and Wilkin, 2006; Han et al., 2010) but it is increasingly clear that in coastal regions the efforts should also be concentrated in improving the model physics (i.e., including non-linear terms, a better representation of the bottom boundary layer and turbulence closure schemes) and the overall spatial resolution (Lyard et al., 2006). In this study we took a step forward in this direction constructing a high resolution non-linear, terrain-following coordinate tidal model of the NPGs.

Although historical observations of tidal elevations and currents in the Patagonian Shelf are too sparse and too short to construct statistical comparisons with the model results, we found a reasonable agreement with the existing observations and previous models. The agreement indicates that the primary physical processes affecting the tides in the NPGs are adequately described by forcing the regional model with data extracted from a global tidal model at the open boundary, without assimilation or tuning of the model parameters (bottom friction and/or horizontal viscosity). The present solutions also revealed additional features of the NPGs circulation poorly delineated or not reported in previous models.

Cotidal charts showed one amphidromic point for the semidiurnal tides off the mouth of SMG and one for the diurnals southeast of VP. The $\mathrm{M}_{2}$ amphidrome have been reported previously but its reproduction relied on some tuning of the bottom friction parameter (i.e., Glorioso and Flather, 1997; Simionato et al., 2004). Additionally, the present simulation have shown small but measurable displacements in the amphidrome's position among constituents of similar class and that no new amphidromes are developed inside the Gulfs. There is a large amplification of the $\mathrm{M}_{2}$ wave after traversing the SMG mouth, a result already reported in low and high resolution models (i.e, Glorioso and Flather, 1997; Moreira et al., 2011). Our results also showed that this amplification extends to all semidiurnals, indicating resonance for the SMG at this frequency. Tidal currents are also larger for the semidiurnal constituents particularly southeast of VP and at the entrance of the three Gulfs. The currents at the diurnal frequency however amplify at the outer shelf due to resonance with continental shelf waves at this frequency (Palma et al., 2004).

At difference with the semidiurnal and diurnal tides the higher harmonics $\mathrm{M}_{4}, \mathrm{MS}_{4}$ and $\mathrm{MN}_{4}$ developed a large amplification inside the NG indicating resonance at quarter-diurnal frequency. Moreira et al. (2011) have also shown a similar but weaker amplification for $\mathrm{M}_{4}$ in NG. The improvement of our solution might be related to the inclusion of these components as open boundary forcing (Davies and Lawrence, 1994). The NPGs show a convergence of tidal energy fluxes and highly localized dissipation areas particularly northeast of VP. In contrast, the higher harmonics showed an export of tidal energy to the shelf. This particular behavior for $\mathrm{M}_{4}$ in the Patagonian shelf has been documented by altimeter studies and high resolution models of the Atlantic Ocean (Ray, 2007; Pairaud et al., 2008). This tendency extends to other higher harmonics like $\mathrm{MS}_{4}$ and $\mathrm{MN}_{4}$.

An additional important result stemming from this study is that the tide can generate residual currents in the NPGs region that are of the same order of magnitude as those generated by winds or offshore forcing (i.e. Palma et al., 2008). We have identified three main patterns of residual circulation: regional coastal currents and gulf-wide gyres; inlet eddies and topographic eddies. Greenberg (1983) and Xue et al. (2000) computed the tidal residuals in the Gulf of Maine-Bay of Fundy region and found a general weak clockwise gyre inside the Gulf, the presence of dipoles (counterrotating eddies) near the head of the Bay and a strong anticlockwise circulation over the major offshore banks (i.e. Georges Bank) generated by tidal rectification mechanisms; results that are consistent with our model results in the NPGs region. Minh et al.
(2014) have analysed the tidal residuals in the Gulf of Tonkin (Yellow Sea) and found an anticlokwise gulf-wide circulation that surrounds Hainan Island and several small scale vortices near the coast. Similarly, Wei et al. (2004) analysed the eulerian and lagrangian tidal residual circulation in the Bohai Sea and found weak currents in the central basin and a pair of intense vortices near the Lyaodong Peninsula.

We have also compared the residual currents generated by forcing the model with $\mathrm{M}_{2}$ and with the full 13 components. The spatial patterns are very similar but the current's intensity is weakened. This result is related to an increase in the bottom friction brought by the additional components and is in agreement with the conclusions of Marinone, (1997). The robustness of the NPGs residual structures was further analysed using vorticity balances and sensitivity experiments. The analysis showed that the intensity of the coastal currents off VP and at the southern and western coasts of SMG as well as the basin-wide gyre in NG were generated through a non-linear tidal rectification mechanism very similar to the one present in straight shelf-slope regions of the open ocean (Pérenne et al., 2000). The physical mechanism relies on the nonlinear advection of local vorticity production, where depth variations and non-linear friction are the major contributors to the current's narrowness and intensity. The structure of the inlet eddies (entrances of NG and SJG) however, are less affected by depth variations. Sensitivity experiments showed that part of the vorticity of these eddies come from shear friction and non-linear advection but their intensity is reinforced by topographic friction effects. A similar mechanism have been invoked in the idealized study of headland eddies by Park and Wang (2000) and the realistic models of Pingree (1978) and Signell and Geyer (1991). The small-scale topographic eddies (hollow and bank) are generated by the nonlinear advection of vorticity generated by topographic mechanisms (bottom torque and topographic differential friction) a result already reported in highly idealized numerical studies of tidal residual circulation (Park and Wang, 1994).

\section{Acknowledgements}

M. H. Tonini and E. D. Palma acknowledge the financial support from CONICET (PIP 1220150100253 and PIP 11220150100253), Agencia Nacional de Promoción Científica y Tecnológica (PICT12-0674) and Universidad Nacional del Sur (24/F053). We also thank Enrique D'onofrio and Martín Saraceno for kindly providing the measured harmonic constants employed in section 3.1. This article strongly benefited from the thoughtful comments of two anonymous reviewers.

\section{References}

Acha, E.M., Mianzan, W.H., Guerrero, R.A., Favero, M. y, Bava, J., 2004. Marine fronts and the continental shelves of austral south America, physical and ecological processes. J. Mar. Syst. 44, 83-105.

Bianchi, A.A., Bianucci, L., Piola, A.R., Pino, D.R., Schloss, I., Poisson, A., Balestrini, C.F., 2005. Vertical stratification and air-sea CO 2 fluxes in the Patagonian shelf. J. Geophys. Res. 110, 1-11.

Chen, C., Beardsley, R.C., Franks, P.J.S., 2001. A 3-D prognostic model study of the ecosystem over Georges Bank and adjacent coastal regions. Part I: physical model. Deep Sea Res. 48, 419-456.

Davies, A.M., Kwong, S.C.M., 2001. Tidal energy fluxes and dissipation on the European continental shelf. J. Geophys. Res. 105, 21969-21989.

Davies, A.M., Lawrence, J., 1994. A three-dimensional model of the M4 tide in the Irish Sea: the importance of open boundary conditions and influence of wind. J. Geophys. Res, 99, 197-227.

Egbert, G.D., Bennett, A.F., Foreman, M.G., 1994. Topex/Poseidon tides estimated using a global inverse model. J. Geophys. Res. 99, 24821-24852.

Foreman, M.G.G., 1977. Manual for Tidal Heights Analysis and Prediction, vols. 77-10. Institute of Ocean Science, Pacific Marine Science Report, Patricia Bay, Sidney, B.C., Canada, p. 97 (unpublished).

Gagliardini, D.A., Rivas, A.L., 2004. Environmental characteristics of San Matías Gulf 
obtained from LANDSAT-TM and ETM+data. Gayana. Concepción 68, 186-193.

Glorioso, P.D., Flather, R.A., 1995. A barotropic model of the currents off SE South America. J. Geophys. Res. 100, 427-440.

Glorioso, P.D., Flather, R.A., 1997. The Patagonian Shelf tides, 1997. Prog. Oceanogr. 40, 263-283.

Glorioso, P.D., Simpson, R.A., 1994. Numerical modelling of the M2 tide on the northern Patagonian Shelf. Cont. Shelf Res. 14, 267-278.

Greenberg, D.A., 1983. Modelling the mean barotropic circulation in the Bay of Fundy and Gulf of Maine. J. Phys. Oceanogr. 13, 886-904.

Han, G., 2000. Three-dimensional modeling of tidal currents and mixing quantities over the Newfoundland Shelf. J. Geophys. Res. 105, 407-422.

Han, G., Paturi, S., de Young, B., Yi, Y., Shum, C.-K., 2010. A 3-D Data-Assimilative tidal Model of the Northwest Atlantic. Atmosphere-Ocean 48, 39-57.

He, R., Wilkin, J.L., 2006. Barotropic tides on the southeast New England shelf: A view from a hybrid data assimilative modeling approach. J. Geophys. Res. 111, $1-20$

Kang, S.K., Lee, S.-R., Lie, H.-J., 1998. Fine Grid Tidal Modeling of the Yellow and East China Seas. Cont. Shelf Res. 18, 739-772.

Klinck, J.M., 1991. Vorticity dynamics of seasonal variations of the Antarctic Circumpolar Current from a modeling study. J. Phys. Oceanogr. 21, 1515-1533. http://dx.doi.org/10.1175/1520-0485(1991)0212.0.co;2.

Lago, L.S., Saraceno, M., Ruiz Etcheverry, L.A., Passaro, M., Oreiro, F., D’Onofrio, E.E. González, R.A., 2017. Improved sea surface height from satellite altimetry in coastal zones: a case study in Southern Patagonia. J. Sel. Topics Appl. Earth Observ. Remote Sens. (under review).

Lyard, F., Lefevre, F., Letellier, T., Francis, O., 2006. Modelling the global ocean tides: modern insights from FES2004. Ocean. Dyn. 56, 394-415.

Marchesiello, P., McWilliams, J.C., Shchepetkin, A., 2001. Open boundary conditions for long-term integration of regional oceanic models. Ocean. Model 3, 1-20.

Marinone, S.G., 1997. Tidal residual currents in the Gulf of California: Is the $\mathrm{M}_{2}$ tidal constituent sufficient to induce them? J. Geophys. Res. 102, 8611-8623.

Mellor, G.L., Yamada, T., 1982. Development of a turbulence closure model for geophysical fluid problems. Rev. Geophys. Space Phys. 20, 851-875.

Minh, N.N., Marchesiello, P., Lyard, F., Ouillon, S., Cambon, G., Allain, D., Dinh, U.V. 2014. Tidal characteristics of the gulf of Tonkin. Cont. Shelf Res. 91, 37-56.

Moreira, D., Simionato, C.G., Dragani, W.C., Nuñez, M.N., 2009. Tidal and residual currents observations at San Matías and San José gulfs, Northen Patagonian, Argentina. J. Coast. Res. 25, 957-968.

Moreira, D., Simionato, C.G., Dragani, 2011. Modeling Ocean Tides and Their Energetics in the North Patagonia Gulfs of Argentina. J. Coast. Res. 27, 87-102.

Nye, J.F., Hajnal, J.V., Hannay, J.H., 1988. Phase saddles and dislocations in twodimensional waves such as the tides. Proc. R. Soc. Lond. 417, 7-20.

Pairaud, I.L., Lyard, F., Auclair, F., Letellier, T., Marsaleix, P., 2008. Dynamics of the semi-diurnal and quarter-diurnal internal tides in the Bay of Biscay. Part 1: Barotropic tides. Cont. Shelf Res. 28, 1294-1315.

Palma, E.D., Matano, R.P., Piola, yA.R., 2004. A numerical study of the Southwestern Atlantic Shelf circulation: Barotropic response to tidal and wind forcing. J. Geophys. Res. 109 (C08014), 1-17. http://dx.doi.org/10.1029/2004JC002315.

Palma, E.D., Matano, R.P., Piola, yA.R., 2008. A numerical study of the Southwestern Atlantic Shelf circulation: Stratified ocean response to local and offshore forcing. J. Geophys. Res. 113 (C11010) http://dx.doi.org/10.1029/2007JC004720.

Park, M.J., Wang, D.P., 1994. Tidal vorticity over isolated topographic features. Cont.
Shelf Res. 14, 1583-1599.

Park, M.J., Wang, D.P., 2000. Tidal vorticity around a coastal promontory. J. Oceanogr. 56, 261-273.

Pérenne, N., Pichon, A., Huet, P., 2000. A numerical study of stratified tidal rectification. Cont. Shelf Res. 20, 37-68.

Pingree, R.D., 1978. The Formation Of The Shambles And Other Banks By Tidal Stirring Of The Seas. J. Mar. Biol. Assoc. U. K. 58, 211-226. http://dx.doi.org/ 10.1017/S0025315400024504.

Pingree, R.D., Maddock, L., 1980. Tidally induced flows around an island due to both frictional and rotational effects. Geophys. J. R. Astronomical Soc. 63, 533-546.

Piola, A.R., Scasso, L.M., 1988. Circulación en el Golfo San Matías. Geoacta 15, 33-51 (in Spanish).

Pisoni, J.P., Rivas, A.L., Piola, A.R., 2014. On the variability of tidal fronts on a macrotidal continental shelf, Northern Patagonia, Argentina. Deep Sea Res. Part I Top. Stud. Oceanogr. 119, 61-68.

Pugh, D.T. 1987. Tides, Surges, and Mean Sea-level. John Wiley \& Sons, Chichester.

Pugh, D.T., Vassie, J.M., 1976. Tide and surge propagation offshore in the Dowsing region of the North Sea. Dtsch. Hydrogr. Zeitschrifi 29, 163-213.

Ray, R.D., 2007. Propagation of the overtide M4 through the deep Atlantic Ocean. Geophys. Res. Lett. 34, 1-4.

Ridderinkhof, H., 1989. Tidal and residual flows in the Western Dutch Wadden Sea: Vorticity balances. Neth. J. Sea Res. 24, 9-16.

Rivas, A.L., 1989. Corrientes originadas por la acción del viento y la marea en el Golfo Nuevo. Geoacta 16, 185-205 (in Spanish).

Rivas, A.L., 1997. Current meter observations in the Argentine continental shelf Cont. Shelf Res. 17, 391-406.

Robinson, I.S., 1983. Tidally induced residual flows. In: Johns, B. (Ed.), Physical Oceanography of Coastal and Shelf Seas. Elsevier, Amsterdam, pp. 321-356.

Romero, S.L., Piola, A.R., Charo, M., García, C.E., 2006. Chlorophyll-a variability off Patagonia based on SeaWiFS data. J. Geophys. Res. 111, 1-11. http://dx.doi.org/ 10.1029/2005JC003244.

Saraceno, M., D'Onofrio, E.E., Fiore, M.E., Grismeyer, W.H., 2010. Tide model comparison over the Southwestern Atlantic Shelf. Cont. Shelf Res. 30, 1865-1875.

Shchepetkin, A.F. McWilliams, J.C.,2005. The regional oceanic modeling system (ROMS): a split-explicit, free-surface, topography-following-coordinate oceanic model. Ocean. Model. 9, 347-404.

Signell, R.P., Geyer, W.R., 1991. Transient eddy formation around headlands J. Geophys. Res. 96, 2561-2575.

Simionato, C.G., Dragani, W., Nuñez, M. y, Engel, M., 2004. A Set of 3-D Nested Models for Tidal Propagation from the Argentinean Continental Shelf to the Río de la Plata Estuary - Part I. M2. J. Coast. Res. 20, 641-936.

Simpson, J.H., Hunter, J.R., 1974. Fronts in the irish sea. Nature 250, 404-406.

Tonini, M.H., Palma, E.D., Piola, A.R., 2013. A numerical study of gyres, thermal fronts and seasonal circulation in austral semi-enclosed gulfs. Cont. Shelf Res. 65, 97-110. http://dx.doi.org/10.1016/j.csr.2013.06.011.

Wei, H., Haainbucher, D., Pohlmann, T., Suendermann, J., 2004. Tidal-induced Lagrangian and Eulerian mean circulation in the Bohai Sea. J. Mar. Syst. 44, $141-151$.

Xue, H., Chai, F., Pettigrew, N.R., 2000. A Model Study of the Seasonal Circulation in the Gulf of Maine. J. Phys. Oceanogr. 30, 1111-1135.

Zimmerman, J.T.F., 1980. Vorticity transfer by tidal currents over an irregular topography. J. Mar. Res. 38, 601-630. 